\title{
Luminosity-dependent evolution of soft X-ray selected AGN
}

\section{New Chandra and XMM-Newton surveys}

\author{
G. Hasinger ${ }^{1}$, T. Miyaji ${ }^{2}$, and M. Schmidt ${ }^{3}$ \\ 1 Max-Planck-Institut für extraterrestrische Physik, Postf. 1312, 85741 Garching, Germany \\ e-mail: ghasinger@mpe.mpg.de \\ 2 Department of Physics, Carnegie Mellon University, 5000 Forbes Avenue, Pittsburg, PA 15213, USA \\ e-mail: miyaji@cmu.edu \\ 3 California Institute of Technology, Pasadena, CA 91125, USA \\ e-mail: mxs@astro.caltech.edu
}

Received 7 October 2004 / Accepted 10 June 2005

\begin{abstract}
We present new results on the cosmological evolution of unabsorbed (type-1) active galactic nuclei (AGN) selected in the soft (0.5-2 keV) X-ray band. From a variety of ROSAT, XMM-Newton and Chandra surveys we selected a total of $\sim 1000$ AGN with an unprecedented spectroscopic and photometric optical/NIR identification completeness. For the first time we are able to derive reliable space densities for low-luminosity (Seyfert-type) X-ray sources at cosmological redshifts. The evolutionary behaviour of AGN shows a strong dependence on X-ray luminosity: while the space density of high-luminosity AGN reaches a peak around $z \sim 2$, similar to that of optically selected QSO, the space density of low-luminosity AGNs peaks at redshifts below $z=1$. This confirms previous ROSAT findings of a luminosity-dependent density evolution. Using a rigorous treatment of the optical identification completeness we are able to show that the space density of AGN with X-ray luminosities $L_{\mathrm{x}}<10^{45} \mathrm{erg} \mathrm{s}^{-1}$ declines significantly towards high redshifts.
\end{abstract}

Key words. galaxies: active - X-rays: general

\section{Introduction}

In recent years the bulk of the extragalactic X-ray background in the $0.1-10 \mathrm{keV}$ band has been resolved into discrete sources with the deepest ROSAT, Chandra and XMM-Newton observations (Hasinger et al. 1998; Mushotzky et al. 2000; Giacconi et al. 2001, 2002; Hasinger et al. 2001; Alexander et al. 2003; Worsley et al. 2004, 2005). Optical identification programmes with Keck (Schmidt et al. 1998; Lehmann et al. 2001; Barger et al. 2001, 2003) and VLT (Szokoly et al. 2004; Fiore et al. 2003) find predominantly unobscured AGN-1 at X-ray fluxes $S_{\mathrm{X}}>10^{-14} \mathrm{erg} \mathrm{cm}^{-2} \mathrm{~s}^{-1}$, and a mixture of unobscured AGN-1 and obscured AGN-2 at fluxes $10^{-14}>S_{\mathrm{X}}>$ $10^{-15.5} \mathrm{erg} \mathrm{cm}^{-2} \mathrm{~s}^{-1}$ with ever fainter and redder optical counterparts, while at even lower X-ray fluxes a new population of star forming galaxies emerges (Hornschemeier et al. 2003; Rosati et al. 2002; Norman et al. 2004). At optical magnitudes $R>24$ these surveys suffer from large spectroscopic incompleteness, but deep optical/NIR photometry can improve the identification completeness significantly, even for the faintest optical counterparts (Zheng et al. 2004; Mainieri et al. 2005).

The AGN/QSO luminosity function and its evolution with cosmic time are key observational quantities for understanding the origin of and accretion history onto supermassive black holes, which are now believed to occupy the centers of most galaxies. X-ray surveys are practically the most efficient means of finding active galactic nuclei (AGNs) over a wide range of luminosity and redshift. Enormous efforts have been made by several groups to follow up X-ray sources with major optical telescopes around the globe, so that now we have fairly complete samples of X-ray selected AGNs. In this work we concentrate on unabsorbed (type-1) AGN selected in the soft $(0.5-2 \mathrm{keV}) \mathrm{X}$-ray band, where due to the previous ROSAT work (see Miyaji et al. 2000, 2001, hereafter Papers I and II) complete samples exist, with sensitivity limits varying over five orders of magnitude in flux, and survey solid angles ranging from the whole high galactic latitude sky to the deepest pencilbeam fields. These samples enable us to construct and probe luminosity functions over cosmological timescales, with an unprecedented accuracy and parameter space.

Conceptually, space densities and luminosity functions are simply derived by dividing the observed number of objects $N$ by the volume $V$, in which they have been surveyed. The binned luminosity function derived in this fashion generally does not represent the center of the bin. In Paper II, we introduced the estimator $N_{\text {obs }} / N_{\text {mdl }}$, where $N_{\text {mdl }}$ is the number of objects 
expected from an analytical representation of the luminosity function. Scaling the model value of the analytical function at the bin center by the estimator removes the binning bias to first order. This method is applied in Sect. 4. A quite different method based on $1 / V_{\max }$ values (Schmidt 1968) for individual objects is used in Sect. 5. It involves a derivation of the zeroredshift luminosity function that is free of binning bias. The luminosity function at higher redshifts is again derived by employing an analytical representation of the luminosity function and scaling it by the ratio of observed over expected numbers. The use of individual $1 / V_{\max }$ values in this case allows accounting for an effective optical magnitude limit beyond which redshifts have generally not been obtained in some surveys.

Throughout this work we use a Hubble constant $H_{0}=$ $70 h_{70} \mathrm{~km} \mathrm{~s}^{-1} \mathrm{Mpc}^{-1}$ and cosmological parameters $\left(\Omega_{\mathrm{m}}, \Omega_{\Lambda}\right)=$ $(0.3,0.7)$ consistent with the WMAP cosmology (Spergel et al. 2003).

\section{The X-ray selected AGN-1 sample}

For the derivation of the X-ray luminosity function and cosmological evolution of AGN we have chosen well-defined fluxlimited samples of active galactic nuclei, with flux limits and survey solid angles ranging over five and six orders of magnitude, respectively. To be able to utilize the massive amount of optical identification work performed previously on a large number of shallow to deep ROSAT surveys, we restricted the analysis to samples selected in the $0.5-2 \mathrm{keV}$ band. In addition to the ROSAT surveys already used in Papers I and II, we included data from the recently published ROSAT North Ecliptic Pole Survey (NEPS, Gioia et al. 2003; Mullis et al. 2004), from an XMM-Newton observation of the Lockman Hole (Mainieri et al. 2002) and the Chandra Deep Fields South (CDF-S, Szokoly et al. 2004; Zheng et al. 2004; Mainieri et al. 2005) and North (CDF-N, Barger et al. 2001, 2003). In order to avoid systematic uncertainties introduced by the varying and a priori unknown AGN absorption column densities we selected only unabsorbed (type-1) AGN, classified by optical and/or X-ray methods. We are using here a definition of type-1 AGN, which is largely based on the presence of broad Balmer emission lines and small Balmer decrement in the optical spectrum of the source (optical type-1 AGN, e.g. the ID classes a, b, and partly c in Schmidt et al. 1998), which largely overlaps the class of X-ray type-1 AGN defined by their X-ray luminosity and unabsorbed X-ray spectrum (Szokoly et al. 2004). However, as Szokoly et al. show, at low X-ray luminosities and intermediate redshifts the optical AGN classification often breaks down because of the dilution of the AGN excess light by the stars in the host galaxy (see e.g. Moran et al. 2002), so that only an X-ray classification scheme can be utilized. Schmidt et al. (1998) have already introduced the X-ray luminosity in their classification. For the deep XMM-Newton and Chandra surveys we in addition use the X-ray hardness ratio to discriminate between X-ray type-1 and type-2 AGN, following Szokoly et al. (2004).

In order to convert the count rates observed in the $0.5-2 \mathrm{keV}$ band to unabsorbed $0.5-2 \mathrm{keV}$ fluxes, we assumed a power law AGN spectrum with a photon index of $\Gamma=2.0$ and Galactic absorption. Typical values of the Galactic neutral hydrogen column density are $(0.5-1) \times 10^{20} \mathrm{~cm}^{-2}$ for the deep surveys and a maximum of $16 \times 10^{20} \mathrm{~cm}^{-2}$ for a small portion of the sky covered by the ROSAT Bright Survey (RBS). Because the band, in which the AGN are selected, is the same as the one for which we calculate the fluxes, systematic differences in the true AGN power law indices have a negligible effect on the derived fluxes. Assuming spectral indices in the range $\Gamma=1-3$, the conversion between the observed $0.5-2 \mathrm{keV}$ count rates and the X-ray flux $S_{\mathrm{x}}$ (here and hereafter, $S_{\mathrm{x}}$ represents the $0.5-2 \mathrm{keV}$ flux and $S_{\mathrm{X} 14}$ is the same quantity measured in units of $10^{-14} \mathrm{erg} \mathrm{s}^{-1} \mathrm{~cm}^{-2}$ ) varies by less than $10 \%$.

The surveys we have used are summarized in Table 1. A total of 944 X-ray selected type-1 AGN were compiled from eight independent samples containing a total of 2566 soft X-ray sources. The number of unidentified sources ${ }^{1}$ in these samples is only 86 (of which 57 could be AGN-1), yielding an unprecedented identification fraction of $97 \%$. Due to the extreme faintness of the optical counterparts, the lowest identification fractions are achieved in the recent deepest samples: $87 \%$ for the XMM-Newton survey in the Lockman Hole and $88 \%$ in the CDF-N. A surprisingly high identification fraction of $98 \%$ has been achieved in the CDF-S through the utilization of photometric redshifts based on extremely faint optical/NIR photometry.

For the computation of the soft X-ray luminosity function SXLF, it is important to define the available survey solid angle as a function of limiting flux. In case there is incompleteness in the spectroscopic identifications in the ROSAT surveys, we have made the usual assumption that the redshift/classification distribution of these unidentified sources is the same as the identified sources at similar fluxes by defining the "effective" survey solid angle as the geometrical survey solid angle multiplied by the completeness of the identifications (see Paper I). This assumption is not correct when the source is unidentified due to non-random causes, in particular its optical faintness. The treatment of this identification incompleteness and the effect of the optical limit on the derived space densities is discussed in detail in Sect. 5.

In several surveys we had to choose an X-ray flux limit a posteriori, based on optical completeness criteria, i.e. maximising the number of optically identified sources, while simultaneously minimising the number of unidentified objects. This procedure can introduce a bias against optically faint sources, if the reason for the missing redshift is the optical faintness of the source and in fields with relatively few objects. We have tried to minimise the impact of this "gerrymandering" effect, e.g. by allowing a number of unidentified sources to enter the sample and then defining the corresponding X-ray flux limits as the geometric mean between the last identified and the next unidentified source. In addition, the wide range for X-ray and thus optical flux limits in our survey tends to reduce biases, which occur at the flux limit of individual surveys.

\footnotetext{
1 We call unidentified sources those, which do not have a reliable redshift determination, either through spectroscopy or through photometric redshifts; however practically all of these have optical or NIR counterparts.
} 
Table 1. The soft X-ray sample.

\begin{tabular}{lccccc}
\hline \hline Survey $^{a}$ & $\begin{array}{c}\text { Solid angle } \\
{\left[\mathrm{deg}^{2}\right]}\end{array}$ & $\begin{array}{c}S_{\mathrm{X} 14, \text { lim }} \\
{[\mathrm{cgs}]}\end{array}$ & $N_{\text {tot }}$ & $N_{\mathrm{AGN}-1}{ }^{b}$ & $N_{\text {unid }}^{c}$ \\
\hline RBS & 20391 & $\approx 250$ & 901 & 203 & 0 \\
SA-N & $684.0-36.0$ & $47.4-13.0$ & 380 & 134 & 5 \\
NEPS & $80.8-70.5$ & $12.4-10.1$ & 252 & 101 & 1 \\
RIXOS & $19.5-15.0$ & $10.2-3.0$ & 340 & 194 & 14 \\
RMS & $0.74-0.32$ & $1.0-0.5$ & 124 & 84 & 7 \\
RDS/XMM & $0.126-0.087$ & $0.38-0.13$ & 81 & 48 & 8 \\
CDF-S & $0.087-0.023$ & $0.022-0.0053$ & 293 & 113 & 1 \\
CDF-N & $0.048-0.0064$ & $0.030-0.0046$ & 195 & 67 & 21 \\
\hline Total & & & 2566 & 944 & 57 \\
\hline
\end{tabular}

a Abbreviations - RBS: the ROSAT Bright Survey (Schwope et al. 2000); SA-N: ROSAT Selected Areas North (Appenzeller et al. 1998); NEPS: ROSAT North Ecliptic Pole Survey (Gioia et al. 2003); RIXOS: ROSAT International X-ray Optical Survey (Mason et al. 2000), RMS: ROSAT Medium Deep Survey, consisting of deep PSPC pointings at the North Ecliptic Pole (Bower et al. 1996), the UK Deep Survey (McHardy et al. 1998), the Marano field (Zamorani et al. 1999) and the outer parts of the Lockman Hole (Schmidt et al. 1998; Lehmann et al. 2000); RDS/XMM: ROSAT Deep Survey in the central part of the Lockman Hole, observed with XMM-Newton (Lehmann et al. 2001; Mainieri et al. 2002; Fadda et al. 2002); CDF-S: the Chandra Deep Field South (Szokoly et al. 2004; Zheng et al. 2004; Mainieri et al. 2005); CDF-N: the Chandra Deep Field North (Barger et al. 2001, 2003).

${ }^{b}$ Excluding AGNs with $z<0.015$.

${ }^{c}$ Objects without redshifts, but hardness ratios consistent with type-1 AGN.

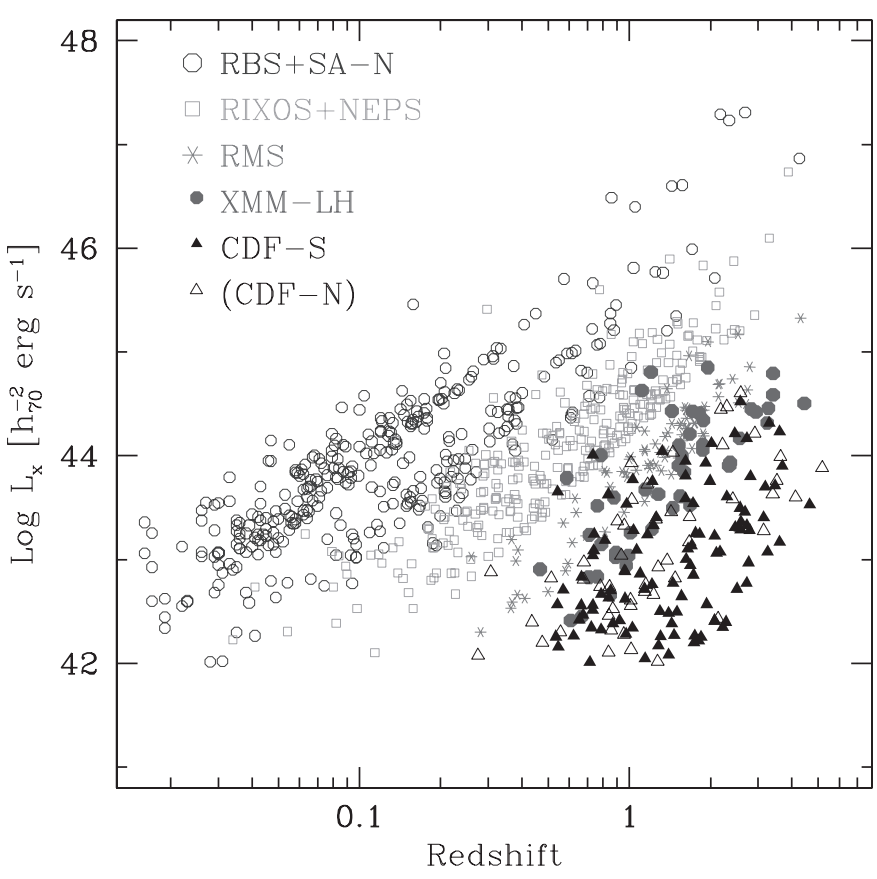

Fig. 1. The AGN-1 soft X-ray sample in the $z-\log L_{\mathrm{x}}$ plane.

The problem of missing redshifts in the faintest surveys is addressed specifically in Sects. 4 and 5.

Below we summarize our sample selection and completeness for each survey. Figure 1 shows the AGN-1 sample in the redshift - luminosity plane. Figure 2 gives the combined solid angle versus flux curve. Both the sample and the solid angle coverage are available in computer readable form under http://mpe.mpg.de/ ghasinger.

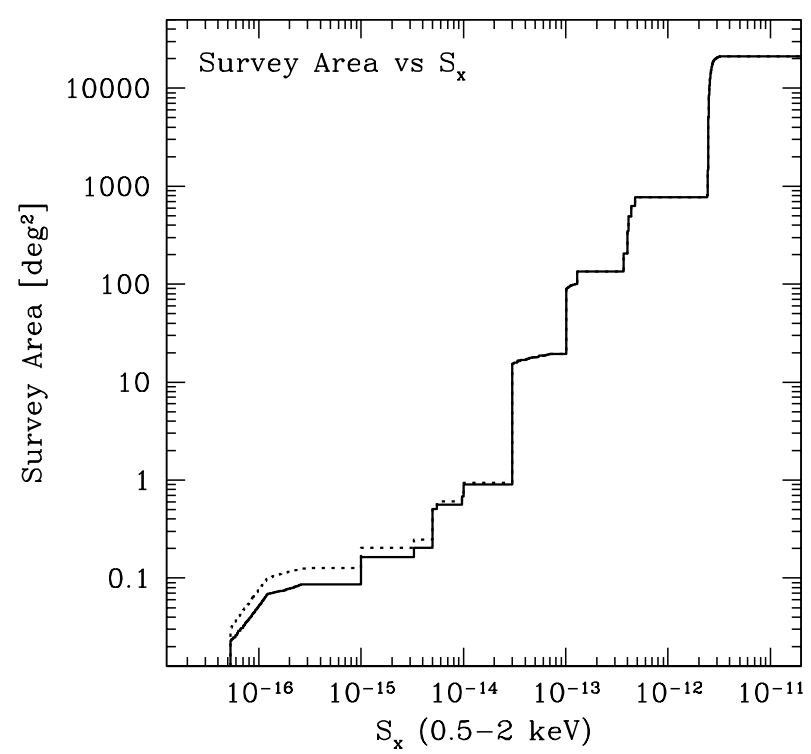

Fig. 2. The survey solid angle of the combined soft X-ray sample as a function of flux. The solid line shows the case where the $\mathrm{CDF}-\mathrm{N}$ sample is excluded as used in Sect. 4. The dotted line includes CDF-N and is used in Sect. 5.

\subsection{The ROSAT Bright Survey (RBS)}

The RBS identified the brightest $\sim 2000$ X-ray sources detected in the ROSAT All-Sky Survey (RASS, Voges et al. 1999) at high galactic latitude, $|b|>30^{\circ}$, excluding the Magellanic Clouds and the Virgo cluster, with ROSAT PSPC count rates above $0.2 \mathrm{~s}^{-1}$. This program achieved a spectroscopic completeness of $99.5 \%$ (Schwope et al. 2000). We selected the sub-sample of 931 sources with count rates above $0.2 \mathrm{~s}^{-1}$ in the ROSAT 0.5-2 keV band (PSPC channels 52-201), which 
is $100 \%$ identified. Since the absorption in our galaxy varies from place to place, the same count rate limit corresponds to different $0.5-2 \mathrm{keV}$ flux limits based on the different galactic $N_{\mathrm{H}}$ values. The $N_{\mathrm{H}}$ value ranges from $(0.5-16) \times 10^{20} \mathrm{~cm}^{-2}$ in the RBS survey area. Correspondingly, the survey solid angle varies steeply with flux from about $3000 \mathrm{deg}^{2}$ at a flux limit of $S_{\mathrm{X} 14}=246$ to a total of $20391 \mathrm{deg}^{2}$ at a flux limit of $S_{\mathrm{X} 14}=360$.

\subsection{The RASS Selected-Area Survey North $(S A-N)$}

This survey gives optical identifications of a representative sample of northern $\left(\delta>-9^{\circ}\right)$ RASS sources in six study areas outside the Galactic plane $\left(|b|>19.6^{\circ}\right)$ with a total of $685 \mathrm{deg}^{2}$. A count rate limited complete RASS subsample comprising 674 sources has been identified (Appenzeller et al. 1998). The fields selected for the survey have a Galactic column density in the range $N_{\mathrm{H}}=(2-11) \times 10^{20} \mathrm{~cm}^{-2}$. We have further selected our sample such that each of the six fields has a complete ROSAT hard-band $(0.5-2 \mathrm{keV}$; channels 52-201) countrate-limited sample with complete identifications $\left(C R_{0.5-2 \mathrm{keV}}>0.01-0.05 \mathrm{cts} \mathrm{s}^{-1}\right)$. To avoid overlap with the RBS, those sources common in both samples were removed from SA-N, yielding a total of 406 sources with $98.5 \%$ spectroscopic completeness.

\subsection{The ROSAT North Ecliptic Pole Survey (NEPS)}

The RASS data in a contiguous area of $80.7 \mathrm{deg}^{2}$ around the North Ecliptic Pole (Galactic latitude $b>29.8^{\circ}$ ) have been used to construct a survey consisting of $445 \mathrm{X}$-ray sources detected above a $4 \sigma$ threshold. Gioia et al. (2003) and Mullis et al. (2004) have identified $99.6 \%$ of these sources and determined redshifts for the extragalactic objects. Since the exposure in the ROSAT All-Sky Survey increases significantly towards the North-Ecliptic Pole, the actual survey sensitivity is a strong function of ecliptic latitude. The original NEPS sample is selected in the full ROSAT PSPC band. For consistency with the other surveys used in our work, we selected sources detected significantly in the PSPC hard band (0.5-2 keV; channels 52-201) by specifying a hard count rate limit as a function of ecliptic latitude. New $0.5-2 \mathrm{keV}$ fluxes were calculated from the hard PSPC count rates in the same way as for the RBS and SA-N objects, taking into account the Galactic neutral hydrogen column density varying in the range $2.6-6.2 \times 10^{20} \mathrm{~cm}^{-2}$ across the NEPS region. Due to the large gradient in exposure times, we have cut the sample to 252 sources with fluxes above $S_{\mathrm{X} 14}=10.1$, where the solid angle of this survey is $70.5 \mathrm{deg}^{2}$, increasing to $80.8 \mathrm{deg}^{2}$ at $S_{\mathrm{X} 14}=12.4$. Only one of these sources remains unidentified in the NEPS sample.

\subsection{The ROSAT International X-ray/Optical Survey (RIXOS)}

The ROSAT International X-ray/Optical Survey (RIXOS, Mason et al. 2000) is a medium-sensitivity survey and optical identification program of X-ray sources discovered in ROSAT high Galactic latitude fields $(|b|>28 \mathrm{deg})$ and observed with the Position Sensitive Proportional Counter (PSPC) detector with a minimum exposure time of $8 \mathrm{ks}$. The survey comprises 82 ROSAT PSPC fields and made use of the central 17 arcmin of each field, however, excluding the target region for pointings on known X-ray sources. The total survey contains 395 X-ray sources, selected in the PSPC $0.5-2 \mathrm{keV}$ band. A flux limit of $S_{\mathrm{X} 14}=3.0$ was adopted for the survey, substantially above the detection threshold of each field, however, the actual spectroscopic completeness limit varies from field to field. We have chosen a strategy, which on one hand maximises the sample of identified AGN-1 and on the other hand minimises the number of unidentified sources. There are 51 fields $\left(12.3 \mathrm{deg}^{2}\right)$ identified completely down to the survey flux limit. Three fields have such a low identification fraction, that we ignore them. For the remaining 28 fields we allow at most one unidentified source. If an unidentified source has the lowest flux of the subsample of a particular field, we exclude this source and raise the flux limit for this field to the geometric average between the flux of this source and that of the last identified source. This way we can define a clean RIXOS sample comprising 340 objects and only 14 unidentified sources, i.e. an identification fraction of $95.9 \%$. The survey solid angle, corrected for spectroscopic incompleteness, rises from $15.0 \mathrm{deg}^{2}$ at $S_{\mathrm{X} 14}=3.0$ to $19.5 \mathrm{deg}^{2}$ at $S_{\mathrm{X} 14}=10.2$.

\subsection{The ROSAT Medium Survey (RMS)}

For this work we have grouped a number of medium-deep ROSAT surveys with flux limits in the range $S_{\mathrm{X} 14}=0.5-1$ into the rms. In particular these comprise pointed observations at the North Ecliptic Pole (Bower et al. 1996), the UK Deep Survey (McHardy et al. 1998), the Marano field (Zamorani et al. 1999) and the outer parts of the Lockman Hole (Schmidt et al. 1998; Lehmann et al. 2000). The North Ecliptic pole pointing covers the same sky area as the center of the NEPS, however, to a flux limit of $S_{\mathrm{X} 14}=1.0$. Again, we remove the overlapping sources between the two surveys. For the UK Deep Survey and the Marano Field we define a flux limit of $S_{\mathrm{X} 14}=0.5$, following Paper I. For the ROSAT PSPC survey of the Lockman Hole we only chose the region not covered by the deeper RDS/XMM survey (see Sect. 2.6) but otherwise selected the completely identified sample with the same flux limits as those chosen for the ROSAT Ultradeep Survey UDS by Lehmann et al. 2001: $S_{\mathrm{X} 14}>0.96$ for PSPC off-axis angles in the range 12.5-18.5 arcmin and $S_{\mathrm{X} 14}>0.55$ for off-axis angles smaller than 12.5 arcmin. Overall, the rms contains 124 sources, at an identification completeness of $94.4 \%$. Correspondingly, the corrected survey solid angle varies in the range $0.30-0.70 \mathrm{deg}^{2}$ for flux limits $S_{\mathrm{X} 14}=0.5-1$.

\subsection{Deep XMM-Newton survey of the Lockman Hole (XMM/RDS)}

The Lockmam Hole (XMM/RDS) has been observed by XMM-Newton a total of 17 times during the $\mathrm{PV}, \mathrm{AO}-1$ and $\mathrm{AO}-2$ phases of the mission, with total good exposure 
times in the range $680-880 \mathrm{ks}$ in the PN and MOS instruments (see Hasinger et al. 2001, 2004 and Worsley et al. 2004 for details). Spectroscopic optical identifications of the ROSAT sources in the LH have been presented by Schmidt et al. (1998) and Lehmann et al. $(2000,2001)$ and a new catalogue from the XMM-Newton PV phase is given in Mainieri et al. (2002). Some photometric redshifts have been discussed in Fadda et al. (2002). Here we selected sources from the $770 \mathrm{ks}$ dataset (Brunner et al. 2005, in prep.) with additional spectroscopic identifications obtained with the DEIMOS spectrograph on the Keck telescope in spring 2003 and 2004 by M. Schmidt and P. Henry (Szokoly et al. 2005, in prep.). In order to maximise the spectroscopic/photometric completeness of the sample, we selected objects in two off-axis intervals: $S_{\mathrm{X} 14}=0.38$ for offaxis angles in the range 10.0-12.5 arcmin and $S_{\mathrm{X} 14}=0.13$ for off-axis angles smaller than 10.0 arcmin. The total number of sources in the XMM/RDS is 81 , with 8 potential AGN-1 still unidentified.

\subsection{Chandra Deep Field South (CDF-S)}

We have used the catalogue of Giacconi et al. (2002) based on the 1 Ms observation of the CDF-S (Rosati et al. 2002). Spectroscopic identifications with the FORS instruments at the ESO VLT have been obtained by Szokoly et al. (2004), yielding a spectroscopic completeness around $60 \%$. Additional spectroscopic redshifts of CDF-S X-ray sources have been obtained with the VIMOS spectrograph at the ESO VLT (Lefevre et al. 2004). The field is also included in the COMBO-17 intermediate-band optical survey, which gives very reliable photometric redshifts for the brighter sources (Wolf et al. 2004). Very deep NIR photometry has been obtained with the ISAAC camera at the VLT in conjunction with deep optical imaging with the HST ACS as part of the GOODS project (Dickinson \& Giavalisco 2003; Mobasher et al. 2004). The CDF-S therefore offers the highest quality photometric redshifts of faint X-ray sources, which are discussed in Zheng et al. (2004) and Mainieri et al. (2005). Using the ISAAC images, tentative photometric redshifts could even be assigned to several of the extreme X-ray/optical sources (EXOs) discussed by Koekemoer et al. (2004). We selected all sources from the Giacconi et al. 2002 catalogue within 10 arcmin from the Chandra pointing center significantly detected in the $0.5-2 \mathrm{keV}$ band. The sample thus contains a total of $293 \mathrm{ob}-$ jects. Combining all spectroscopic and photometric redshifts, only 2 sources in the CDFS remain unidentified, of which one could be an AGN-1. The survey solid angle for the CDF-S has been estimated using a simple off-axis dependent flux limit. The solid angle increases from $0.023 \mathrm{deg}^{2}$ at $S_{\mathrm{X} 14}=0.0053$ to $0.087 \mathrm{deg}^{2}$ at $S_{\mathrm{X} 14}=0.027$.

\subsection{Chandra Deep Field North (CDF-N)}

We have used selected X-ray sources from the $2 \mathrm{Ms}$ CDF-N source catalogue by Alexander et al. (2003) along with optical identifications by Barger et al. (2003) for our AGN-1 sample. Following Szokoly et al. (2004), we selected AGN-1 either from broad permitted Balmer lines or from the X-ray luminosity and hardness, using HR $<-0.2$. We set our flux limits such that we also have sufficient hard (2-8 keV) sensitivity to exclude objects with $\mathrm{HR}>-0.2$ and to include as many sources as possible which meet these criteria. Unlike the $\mathrm{CDF}-\mathrm{S}$, the CDF-N exposure map has a complicated structure and a simple off-axis dependence of the limiting flux is not a good approximation. Thus we have used the rectangular region of $170 \mathrm{arcmin}^{2}$, which has the deepest coverage and is mostly co-spatial with the region covered by HST ACS in the GOODS project (Giavalisco et al. 2004; Cowie et al. 2004). Within this region, the exposure and background are smooth enough that the photon counts limit of the detected sources can be approximated by a simple function of off-axis angle. In practice, due to statistical fluctuations, three sources have upper limits to HR between -0.1 and -0.2 and those have been considered to meet our hardness ratio criterion. Among the 128 sources meeting the soft counts limit and hardness ratio criteria, 20 are unidentified and 5 are stars (85\% completeness). Only one broad-line AGN had a harder hardness ratio than our limit; this was also included in our type-1 AGN sample. The flux-solid angle relation has been calculated from the "limiting flux map", where the counts limit is divided by the soft-band exposure map (in seconds) and multiplied by the conversion factor of $5 \times 10^{-12} \mathrm{erg} \mathrm{s}^{-1} \mathrm{~cm}^{-2}$ (Alexander et al. 2003). Due to the incompleteness in this field, where most unidentified sources are optically faint, this sample has not been included in the analysis in Sect. 4, but considered in Sect. 5, where a method is developed to account for the optical magnitude limit in calculating the survey volume.

\section{Number counts for different source classes}

The combination of a large number of surveys with a wide range of sensitivity limits and solid angle coverage presents a unique resource. On one hand, the surveys presented here resolve the soft X-ray background almost completely. On the other hand, we have an almost complete optical identification and redshift determination for all constituents. For the first time in any astrophysical waveband we are thus in the position to study the complete contribution of different object classes to the X-ray background and their evolution with cosmic time. Using the solid angle versus flux limit curve given in Fig. 2 we compiled number counts for the total sample including all classes of sources and for the subclass of AGN-1. Figure 3 (top) shows the cumulative source counts. For clarity we also show normalized differential source counts $\mathrm{d} N / \mathrm{d} S_{\mathrm{X} 14} S_{\mathrm{X} 14}^{2.5}$ in the bottom panel of Fig. 3. Euclidean source counts would correspond to horizontal lines in this graph.

For the total source counts, the well-known broken power law behaviour is confirmed with high precision. We fitted a broken power law to the differential source counts and obtain power law indices of $\alpha_{\mathrm{b}}=2.34 \pm 0.01$ and $\alpha_{\mathrm{f}}=1.55 \pm 0.04$ for the bright and faint end, respectively, a break flux of $S_{\mathrm{X} 14}=$ $0.65 \pm 0.10$ and a normalisation of $\mathrm{d} N / \mathrm{d} S_{\mathrm{X} 14}=103.5 \pm 5.3 \mathrm{deg}^{-2}$ at $S_{\mathrm{X} 14}=1.0$ with a reduced $\chi^{2}=1.51$. The total differential source counts, normalized to a Euclidean behaviour $\left(\mathrm{d} N / \mathrm{d} S_{\mathrm{X} 14} \times S_{\mathrm{X} 14}^{2.5}\right)$ is shown with open symbols in Fig. 3. We 

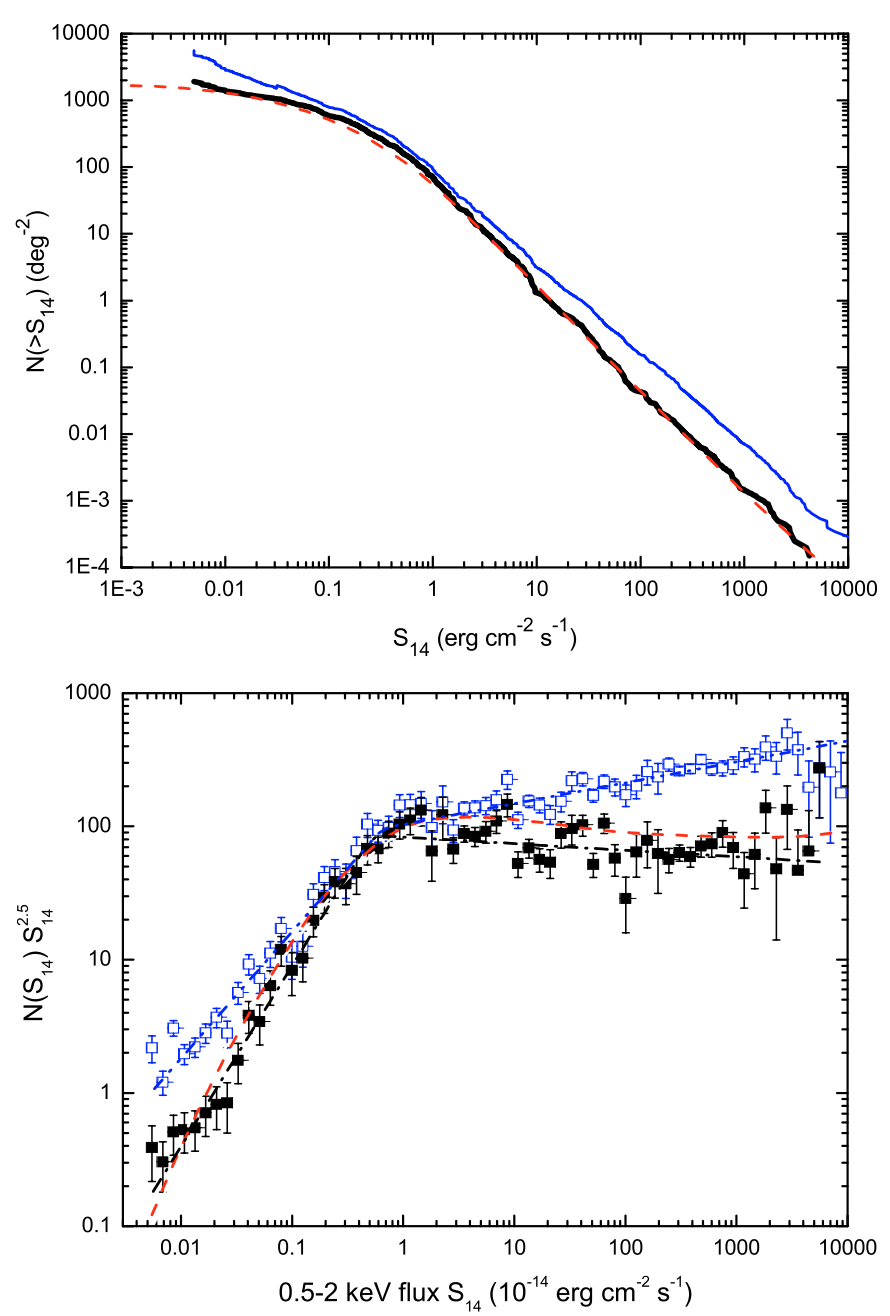

Fig. 3. a) Cumulative number counts $N(>S)$ for the total sample (upper thin line) and the AGN-1 subsample (lower thick line). b) Differential number counts of the total sample of X-ray sources (open squares) and the AGN-1 subsample (filled squares). The dot-dashed lines refer to broken powerlaw fits to the differential source counts (see text). The dashed red line shows the prediction for type-1 AGN based on the model described in in Sect. 5.

see that the total source counts at bright fluxes, as determined by the ROSAT All-Sky Survey data, are significantly flatter than Euclidean, consistent with the discussion in Hasinger et al. (1993). Moretti et al. (2003), on the other hand, have derived a significantly steeper bright flux slope $\left(\alpha_{\mathrm{b}} \approx 2.8\right)$ from ROSAT HRI pointed observations. This discrepancy can probably be attributed to the selection bias against bright sources, when using pointed observations where the target area has to be excised.

Type-1 AGN are the most abundant population of soft $\mathrm{X}$-ray sources. For the determination of the AGN-1 number counts we include those unidentified sources, which have hardness ratios consistent with AGN-1 (a contribution of $\sim 6 \%$, see Table 1). Figure 3 shows, that the break in the total source counts at intermediate fluxes is produced by type-1 AGN, which are the dominant population there. Both at bright fluxes and at the faintest fluxes, type-1 AGN contribute about $30 \%$ of the X-ray source population. At bright fluxes, they have to share with clusters, stars and BL-Lac objects, at faint fluxes they compete with type-2 AGN and normal galaxies. We fitted a broken power law to the differential AGN-1 source counts and obtain power law indices of $\alpha_{\mathrm{b}}=2.55 \pm 0.02$ and $\alpha_{\mathrm{f}}=1.15 \pm 0.05$ for the bright and faint end, respectively, a break flux of $S_{\mathrm{X} 14}=0.53 \pm 0.05$, consistent with that of the total source counts within errors, and a normalisation of $\mathrm{d} N / \mathrm{d} S_{\mathrm{X} 14}=83.2 \pm 5.5 \mathrm{deg}^{-2}$ at $S_{\mathrm{X} 14}=1.0$ with a reduced $\chi^{2}=1.26$. The AGN-1 differential source counts, normalized to a Euclidean behaviour $\left(\mathrm{d} N / \mathrm{d} S_{\mathrm{X} 14} \times S_{\mathrm{X} 14}^{2.5}\right)$ is shown with filled symbols in Fig. 3. Also shown is the prediction of the best-fit SXLF model discussed in Sect. 5.

\section{The SXLF and the space density function}

\subsection{Basic method}

In this section, we present the binned Soft X-ray Luminosity Function (SXLF) of type-1 AGNs. The basic approach is to use the $N^{\text {obs }} / N^{\mathrm{mdl}}$ estimator described in Paper II. The procedure is outlined below:

1. Divide the combined sample into several redshift shells. For each redshift shell, fit the AGN XLF with a smooth analytical function using a Maximum-likelihood fit over each object (i.e., without binning; see Paper I for details).

2. For the fitted model in each redshift shell, check the absolute goodness of fit with one- and twodimensional Kolmogorov-Smirnov tests (hereafter, 1D-KS and 2D-KS tests respectively; Press et al. 1992; Fassano \& Franceschini 1987). The K-S tests are also for the unbinned data sets and thus are free from artefacts and biases due to binning.

3. For each redshift shell, bin the objects in luminosity bins to determine the observed number of objects $\left(N^{\text {obs }}\right)$.

4. For each luminosity bin, evaluate the analytical fit at the central luminosity/redshift $\left(\mathrm{d} \Phi^{\mathrm{mdl}} / \mathrm{d} \log L_{\mathrm{x}}\right)$.

5. Calculate the predicted number of AGNs in the bin $\left(N^{\mathrm{mdl}}\right)$.

6. The final result is

$$
\mathrm{d} \Phi / \mathrm{d} \log L_{\mathrm{x}}=\mathrm{d} \Phi^{\mathrm{mdl}} / \mathrm{d} \log L_{\mathrm{x}} \cdot N^{\mathrm{obs}} / N^{\mathrm{mdl}} .
$$

For the analytical expression of the SXLF in each redshift shell, we use the smoothed two power law formula. Because the redshift shells have a finite widths, the fit results depend on the evolution of the SXLF within them:

$$
\frac{\mathrm{d} \Phi\left(L_{\mathrm{x}}, z\right)}{\mathrm{d} \log L_{\mathrm{x}}} \propto\left[\left(\frac{L_{\mathrm{x}}}{L_{\mathrm{x}, *}}\right)^{\gamma_{1}}+\left(\frac{L_{\mathrm{x}}}{L_{\mathrm{x}, *}}\right)^{\gamma_{2}}\right]^{-1} \cdot e_{\mathrm{d}}\left(z, L_{\mathrm{x}}\right),
$$

where $e_{\mathrm{d}}\left(z, L_{\mathrm{x}}\right)$ is the density evolution factor. While the final results are insensitive to the detailed behavior of $e_{\mathrm{d}}\left(z, L_{\mathrm{x}}\right)$ within the shell at most locations in the $\left(L_{\mathrm{x}}, z\right)$ space, we have taken our best-estimate by using the luminosity-dependent density evolution (LDDE) model derived later in Sect. 4.4. The luminosity range of the fit is from $\log L_{\mathrm{x}}=42.0$ to the maximum available luminosity in the sample.

In this section, we tried to make the sample as complete as possible, and we excluded the $\mathrm{CDF}-\mathrm{N}$ from the analysis, where the incompleteness fraction is significant and most of the unidentified sources are optically faint. All of the unidentified sources in the ROSAT samples are optically bright and 
Table 2. Best-fit parameters for each redshift shell.

\begin{tabular}{ccrccccc}
\hline \hline$z$-range & $z_{c}$ & $N$ & $A_{44}^{a}$ & $\log L_{\mathrm{x}, *}^{a}$ & $\gamma_{1}$ & $\gamma_{2}$ & $\mathrm{KS}_{-\mathrm{prob}}{ }^{b}$ \\
\hline $0.0-0.2$ & 0.1 & 268 & $(3.64 \pm 0.22) \times 10^{-7}$ & $43.45_{-0.27}^{+0.32}$ & $0.35_{-0.40}^{+0.31}$ & $2.1_{-0.3}^{+0.4}$ & $0.84,0.17,0.14$ \\
$0.2-0.4$ & 0.3 & 139 & $(9.76 \pm 0.83) \times 10^{-7}$ & $43.91_{-0.36}^{+0.38}$ & $0.70_{-0.45}^{+0.31}$ & $2.5_{-0.3}^{+0.5}$ & $0.97,0.71,0.65$ \\
$0.4-0.8$ & 0.6 & 143 & $(1.84 \pm 0.15) \times 10^{-6}$ & $43.91_{-0.41}^{+0.41}$ & $0.84_{-0.32}^{+0.23}$ & $2.3_{-0.2}^{+0.3}$ & $1.00,0.03,0.08$ \\
$0.8-1.6$ & 1.2 & 187 & $(1.19 \pm 0.09) \times 10^{-5}$ & $43.97_{-0.13}^{+0.13}$ & $0.10_{-0.19}^{+0.17}$ & $2.3_{-0.1}^{+0.2}$ & $0.91,0.68,0.88$ \\
$1.6-3.2$ & 2.4 & 110 & $(1.03 \pm 0.10) \times 10^{-5}$ & $44.39_{-0.17}^{+0.16}$ & $0.15_{-0.18}^{+0.16}$ & $2.3_{-0.2}^{+0.2}$ & $0.91,0.55,0.44$ \\
$3.2-4.8$ & 4.0 & 17 & $(4.53 \pm 1.10) \times 10^{-6}$ & $44.43_{-0.37}^{+0.40}$ & $-0.26_{-0.75}^{+0.56}$ & $1.9_{-0.3}^{+0.4}$ & $0.95,0.21,0.39$ \\
\hline
\end{tabular}

Parameter values which have been fixed during the fit are labelled by $(*){ }^{a}$ Units $-A_{44}: h_{70}^{3} \mathrm{Mpc}^{-3}, L_{\mathrm{x}, *}: 10^{44} h_{70}^{-2} \mathrm{erg} \mathrm{s}^{-1}$. $^{b}$ The three values are probabilities in two 1D-KS test for the distribution, $L_{\mathrm{x}}, 1 \mathrm{D}-\mathrm{KS}$ test for the $z$ distribution and the 2D-KS test for the $\left(L_{\mathrm{x}}, z\right)$ space respectively.

the reasons for them to be unidentified are mostly by random causes, i.e., are not correlated with the intrinsic properties of the source. For the CDF-S, extensive photometric redshift studies including COMBO-17 (Wolf et al. 2004) and a careful individual photometric redshift determination of X-ray sources by Zheng et al. (2004) and Mainieri et al. (2005) has left only one potential AGN-1 without redshift information. For RDS/XMM, 2 of the 8 unidentified sources which could be type-1 AGNs from X-ray hardness/spectra criteria are optically bright and they have remained unidentified so far for random reasons. The remaining 6 are optically faint $(R \geq 24.0)$ and the reason for remaining unidentified may well be correlated with redshift.

As our nominal case, we took the first-order approach and defined "effective" survey solid angle (as a function of flux), which is the geometrical survey solid angle multiplied by the completeness, i.e. the fraction of identified X-ray sources in the survey, whether or not they are optically faint or bright. The correction has been made in each survey. In addition, we have also considered the upper bounds on the binned SXLF and the space density function from the sample where all the unidentified optically faint $(R \geq 24.0)$ sources in turn are assigned the central redshift of each redshift shell.

In the latter case, we used the geometrical solid angle for the CDF-S and the incompleteness correction to the RDS/XMM solid angle was only for the optically bright $R<24$ unidentified sources.

\subsection{The binned SXLF}

The best fit parameters of Eq. (2) for each redshift shell are shown in Table 2 along with the results of the 1D- and $2 \mathrm{D}-\mathrm{KS}$ tests (see the notes of the table). The normalization is defined by:

$A_{44}=\frac{\mathrm{d} \Phi\left(L_{\mathrm{x}}=10^{44} \operatorname{erg~s}^{-1}, z=z_{\mathrm{c}}\right)}{\mathrm{d} \log L_{\mathrm{x}}}$,

where $z_{\mathrm{c}}$ is the central redshift of the shell. The parameter errors in Table 2 correspond to a likelihood change of 2.7 (90\% confidence errors), except for the normalization $A_{44}$, which cannot be a fit parameter in the maximum-likelihood method. The errors of $A_{44}$ are simply taken as the $90 \%$ Poisson errors of the number of the sources. Defining the normalization at a fixed luminosity $\left(\log L_{\mathrm{x}}=44\right)$ minimizes its dependence on other parameters. In any case, Eq. (2) gives a statistically satisfactory expression for all redshift shells as shown in Table 2.

We have made luminosity bins starting with a minimum luminosity of $\log L_{\mathrm{x}}=42.0$ with a smallest bin size of $\Delta \log L_{\mathrm{x}}=0.25 \mathrm{in}$ each redshift shell. If there are fewer than 10 AGNs in a bin, we have further rebinned up to a maximum bin size of $\Delta \log L_{\mathrm{x}}=1.0$. Table 3 shows the full binned results for the nominal case, along with observed number of AGNs $\left(N^{\mathrm{obs}}\right)$, model (Table 2) predicted number $\left(N^{\mathrm{mdl}}\right)$ and final estimated values of the SXLF at the center of each bin. For reference, the additional number $N^{\mathrm{f}}$ of AGNs for the case where all the optically-faint unidentified sources are assigned the central redshift of the bin (in duplicate, as described above) are also shown in the last column of Table 3. The full SXLF in the 6 redshift shells is plotted in Fig. 4 in separate panels. In all but the closest redshift shell panel, the best-fit two power law function to the $0.015<z<0.2$ SXLF (Table 2) are also overplotted for reference. Three overall analytical expressions discussed in Sect. 4.4 are also overplotted for comparison as discussed there. Because of the high completeness of our sample, the redshift distribution of the optically faint sources affects the final SXLF results very little except for the case where all of them happen to fall into the highest redshift shell. In this case, the SXLF in the $3.2<z<4.8,44<\log L_{\mathrm{x}}$ bin almost double. This is also verified by a comparison with the alternative approach outlined in Sect. 5.

\subsection{Evolution of the space density}

In this section, we investigate the evolution of the type-1 AGN space density in different luminosity classes as a function of redshift. The estimator of the space density is the $N^{\mathrm{obs}} / N^{\mathrm{mdl}}$. The fit with Eq. (2) has been made in finer redshift shells than in Sect. 4.2. The space densities as a function of redshift were calculated in five luminosity classes with $\log L_{\mathrm{x}}$ of 42-43, 43-44, $44-45,45-46$, and $>46$ as well as the sum over all luminosities with $\log L_{\mathrm{x}}>42$. The resulting curves are shown in Fig. 5a for the nominal calculations. The incompleteness upper bounds have also been calculated, but have not been shown here for the visibility of the figure. These upper bounds are shown in Sect. 6 (Figs. 11 and 13). Since the Black Hole growth function is more closely linked to the emissivity per comoving volume, we also show the emissivity as a function of redshift in the same luminosity classes in Fig. 5b. 
Table 3. The full binned SXLF values.

\begin{tabular}{|c|c|c|c|c|c|}
\hline$z$ & $\log L_{\mathrm{x}}{ }^{a}$ & $N^{\mathrm{obs}}$ & $N^{\mathrm{mdl}}$ & ${\frac{\mathrm{d} \Phi}{\mathrm{d} \log L_{\mathrm{x}}}}^{b}$ & $\left(N^{\mathrm{f}}\right)$ \\
\hline $0.015-0.2$ & $42.00-42.50$ & 10 & 11.5 & $\left(1.3_{-04}^{+0.5}\right) \times 10^{-5}$ & \\
\hline $0.015-0.2$ & $42.50-42.75$ & 15 & 15.3 & $\left(1.0_{-03}^{+0.3}\right) \times 10^{-5}$ & \\
\hline $0.015-0.2$ & $42.75-43.00$ & 20 & 25.2 & $\left(6.4_{-1.4}^{+1.7}\right) \times 10^{-6}$ & \\
\hline $0.015-0.2$ & $43.00-43.25$ & 48 & 38.6 & $\left(7.1_{-1.0}^{+1.1}\right) \times 10^{-6}$ & \\
\hline $0.015-0.2$ & $43.25-43.50$ & 45 & 44.9 & $\left(3.4_{-0.5}^{+0.6}\right) \times 10^{-6}$ & \\
\hline $0.015-0.2$ & $43.50-43.75$ & 38 & 45.7 & $\left(1.4_{-0.2}^{+0.2}\right) \times 10^{-6}$ & \\
\hline $0.015-0.2$ & $43.75-44.00$ & 36 & 34.6 & $\left(6.5_{-1.1}^{+1.2}\right) \times 10^{-7}$ & \\
\hline $0.015-0.2$ & $44.00-44.25$ & 27 & 23.7 & $\left(2.4_{-0.5}^{+0.5}\right) \times 10^{-7}$ & \\
\hline $0.015-0.2$ & $44.25-44.50$ & 22 & 16.9 & $\left(8.5_{-1.8}^{+2.1}\right) \times 10^{-8}$ & \\
\hline $0.015-0.2$ & $44.50-45.50$ & 7 & 11.2 & $\left(2.1_{-0.8}^{+1.1}\right) \times 10^{-9}$ & \\
\hline $0.2-0.4$ & $42.25-43.00$ & 9 & 9.6 & $\left(2.0_{-0.7}^{+0.9}\right) \times 10^{-5}$ & \\
\hline $0.2-0.4$ & $43.00-43.25$ & 12 & 11.2 & $\left(10.0_{-2.8}^{+3.6}\right) \times 10^{-6}$ & \\
\hline $0.2-0.4$ & $43.25-43.50$ & 23 & 19.1 & $\left(7.0_{-1.5}^{+1.7}\right) \times 10^{-6}$ & \\
\hline $0.2-0.4$ & $43.50-43.75$ & 22 & 24.1 & $\left(3.0_{-0.6}^{+0.8}\right) \times 10^{-6}$ & \\
\hline $0.2-0.4$ & $43.75-44.00$ & 25 & 25.7 & $\left(1.5_{-0.3}^{+0.4}\right) \times 10^{-6}$ & \\
\hline $0.2-0.4$ & $44.00-44.25$ & 15 & 18.1 & $\left(4.7_{-1.2}^{+1.5}\right) \times 10^{-7}$ & \\
\hline $0.2-0.4$ & $44.25-44.50$ & 11 & 10.6 & $\left(1.8_{-0.5}^{+0.7}\right) \times 10^{-7}$ & \\
\hline $0.2-0.4$ & $44.50-44.75$ & 11 & 7.6 & $\left(6.4_{-1.9}^{+2.4}\right) \times 10^{-8}$ & \\
\hline $0.2-0.4$ & $44.75-45.50$ & 11 & 11.8 & $\left(2.5_{-0.7}^{+0.9}\right) \times 10^{-9}$ & \\
\hline $0.2-0.4$ & $45.50-46.50$ & 0 & 0.4 & $<9.9 \times 10^{-11}$ & \\
\hline $0.4-0.8$ & $42.00-42.75$ & 18 & 17.1 & $\left(1.1_{-0.2}^{+0.3}\right) \times 10^{-4}$ & (6) \\
\hline $0.4-0.8$ & $42.75-43.50$ & 23 & 22.0 & $\left(2.3_{-0.5}^{+0.6}\right) \times 10^{-5}$ & \\
\hline $0.4-0.8$ & $43.50-43.75$ & 13 & 15.4 & $\left(5.5_{-15}^{+1.9}\right) \times 10^{-6}$ & \\
\hline $0.4-0.8$ & $43.75-44.00$ & 21 & 23.4 & $\left(2.6_{-0.6}^{+0.7}\right) \times 10^{-6}$ & \\
\hline $0.4-0.8$ & $44.00-44.25$ & 26 & 22.7 & $\left(1.3_{-0.2}^{+0.03}\right) \times 10^{-6}$ & \\
\hline $0.4-0.8$ & $44.25-44.50$ & 13 & 16.0 & $\left(2.9_{-0.8}^{+1.0}\right) \times 10^{-7}$ & \\
\hline $0.4-0.8$ & $44.50-44.75$ & 11 & 11.1 & $\left(1.0_{-0.3}^{+0.4}\right) \times 10^{-7}$ & \\
\hline $0.4-0.8$ & $44.75-45.25$ & 13 & 8.8 & $\left(2.2_{-0.6}^{+0.7}\right) \times 10^{-8}$ & \\
\hline $0.4-0.8$ & $45.25-46.25$ & 5 & 6.2 & $\left(2.2_{-1.0}^{+1.4}\right) \times 10^{-10}$ & \\
\hline $0.4-0.8$ & $46.25-47.25$ & 0 & 0.2 & $<1.8 \times 10^{-11}$ & \\
\hline $0.8-1.6$ & $42.00-42.50$ & 12 & 11.0 & $\left(4.2_{-1.2}^{+1.5}\right) \times 10^{-5}$ & (1) \\
\hline $0.8-1.6$ & $42.50-43.00$ & 13 & 12.4 & $\left(3.6_{-1.0}^{+1.2}\right) \times 10^{-5}$ & \\
\hline $0.8-1.6$ & $43.00-43.50$ & 14 & 17.7 & $\left(2.4_{-0.6}^{+0.0}\right) \times 10^{-5}$ & (6) \\
\hline $0.8-1.6$ & $43.50-43.75$ & 12 & 12.9 & $\left(2.2_{-0.6}^{+0.6}\right) \times 10^{-5}$ & \\
\hline $0.8-1.6$ & $43.75-44.00$ & 20 & 16.4 & $\left(2.0_{-0.4}^{+0.5}\right) \times 10^{-5}$ & \\
\hline $0.8-1.6$ & $44.00-44.25$ & 27 & 25.5 & $\left(8.2_{-1.6}^{+1.8}\right) \times 10^{-6}$ & \\
\hline $0.8-1.6$ & $44.25-44.50$ & 27 & 25.0 & $\left(2.8_{-0.5}^{+0.0}\right) \times 10^{-6}$ & \\
\hline $0.8-1.6$ & $44.50-44.75$ & 26 & 25.7 & $\left(7.5_{-1.5}^{+1.7}\right) \times 10^{-7}$ & \\
\hline $0.8-1.6$ & $44.75-45.00$ & 13 & 16.7 & $\left(1.5_{-0.4}^{+0.5}\right) \times 10^{-7}$ & \\
\hline $0.8-1.6$ & $45.00-45.75$ & 15 & 18.5 & $\left(1.1_{-0.3}^{+0.3}\right) \times 10^{-8}$ & \\
\hline $0.8-1.6$ & $45.75-46.75$ & 8 & 4.4 & $\left(2.2_{-0.8}^{+1.0}\right) \times 10^{-10}$ & \\
\hline $0.8-1.6$ & $46.75-47.75$ & 0 & 0.3 & $<4.0 \times 10^{-12}$ & \\
\hline $1.6-3.2$ & $42.00-42.75$ & 10 & 9.5 & $\left(2.2_{-0.7}^{+0.9}\right) \times 10^{-5}$ & \\
\hline $1.6-3.2$ & $42.75-43.25$ & 11 & 13.1 & $\left(1.4_{-0.4}^{+0.5}\right) \times 10^{-5}$ & (1) \\
\hline $1.6-3.2$ & $43.25-44.00$ & 23 & 22.0 & $\left(1.4_{-0.3}^{+0.3}\right) \times 10^{-5}$ & (6) \\
\hline $1.6-3.2$ & $44.00-44.50$ & 27 & 23.0 & $\left(8.4_{-1.6}^{+1.9}\right) \times 10^{-6}$ & \\
\hline $1.6-3.2$ & $44.50-45.00$ & 16 & 16.9 & $\left(1.2_{-0.3}^{+0.4}\right) \times 10^{-6}$ & \\
\hline $1.6-3.2$ & $45.00-45.25$ & 12 & 8.4 & $\left(2.9_{-0.8}^{+1.0}\right) \times 10^{-7}$ & \\
\hline $1.6-3.2$ & $45.25-46.00$ & 8 & 13.1 & $\left(9.1_{-3.1}^{+4.2}\right) \times 10^{-9}$ & \\
\hline $1.6-3.2$ & $46.00-47.00$ & 0 & 2.9 & $<1.2 \times 10^{-10}$ & \\
\hline $1.6-3.2$ & $47.00-48.00$ & 3 & 0.5 & $\left(4.6_{-2.5}^{+4.2}\right) \times 10^{-12}$ & \\
\hline $3.2-4.8$ & $43.00-44.00$ & 5 & 4.9 & $\left(3.8_{-1.6}^{+2.4}\right) \times 10^{-6}$ & (1) \\
\hline $3.2-4.8$ & $44.00-45.00$ & 8 & 6.9 & $\left(3.2_{-1.1}^{+1.5}\right) \times 10^{-6}$ & (6) \\
\hline $3.2-4.8$ & $45.00-46.00$ & 1 & 3.2 & $\left(1.7_{-1.4}^{+3.5}\right) \times 10^{-8}$ & \\
\hline $3.2-4.8$ & $46.00-47.00$ & 3 & 1.9 & $\left(9.8_{-5.3}^{+1.8}\right) \times 10^{-10}$ & \\
\hline $3.2-4.8$ & $47.00-48.00$ & 0 & 0.6 & $<2.9 \times 10^{-11}$ & \\
\hline
\end{tabular}

Notes: ${ }^{a} L_{\mathrm{x}} h_{70}^{-2} \mathrm{erg} \mathrm{s}^{-1}$ in the $0.5-2 \mathrm{keV}$ band. ${ }^{b} h_{70}^{3} \mathrm{Mpc}^{-3}$; the quoted errors are $68 \%$ Poisson errors using approximations by Gehrels (1986) of the number of AGNs.
Figure 5a clearly shows a shift of the number density peak with luminosity, in the sense that more luminous AGNs (QSOs) peak earlier in the history of the universe, while the low luminosity ones arise later. Also, there is a clear decline of the derived space densities at least for luminosities of $\log L_{\mathrm{x}}<44$, even when the optical incompleteness upper bounds are taken into account. The counting statistics and spectroscopic incompleteness for the more luminous AGNs do not allow to determine a decline, but do also not exclude it. This issue is further discussed in Sects. 5 and 6.

In order to show the behaviour of the luminosity dependence of the evolution more quantitatively, we have also made a maximum-likelihood fit of the evolution curve in each of the luminosity bins, with $\log L_{\mathrm{x}}$ ranges of 42-43, 43-44, 44-45, and 45-46. We used two power law components of the $(1+z)$ evolution with a cutoff redshift:

$e_{\mathrm{d}}\left(z, L_{\mathrm{xc}}\right)=\left\{\begin{array}{ll}(1+z)^{p 1} & \left(z \leq z_{\mathrm{c}}\right) \\ e_{\mathrm{d}}\left(z_{\mathrm{c}}\right)\left[(1+z) /\left(1+z_{\mathrm{c}}\right)\right]^{p 2} & \left(z>z_{\mathrm{c}}\right)\end{array}\right.$,

where $L_{\mathrm{xc}}$ is the central (logarithmic) luminosity of the bin. As was the case for Eq. (2), the fit depends on the shape of the luminosity function (along the luminosity direction) within the luminosity bin. Again, we have fixed the behavior in the luminosity direction using those from the LDDE model (Sect. 4.4) as a template. We also show the normalization:

$A_{0} \equiv \mathrm{d} \Phi\left(L_{\mathrm{x}}=L_{\mathrm{xc}}, z=0\right) / \mathrm{d} \log L_{\mathrm{x}}$.

The best-fit results are shown in Table 4 together with the $\mathrm{K}-\mathrm{S}$ probabilities. In the $\log L_{\mathrm{x}}=45-46$ bin, the fit for $p_{2}$ was unconstrained. Thus we have fixed the values of $p_{2}$ to that from LDDE (see below) for this luminosity bin.

\subsection{Global representations by analytical functions}

It is sometimes useful to provide a simple analytical fit for the SXLF over the whole redshift-luminosity range. We first used the pure-luminosity evolution (PLE) form, in order to enable a comparison with previous work:

$\frac{\mathrm{d} \Phi\left(L_{\mathrm{x}}, z\right)}{\mathrm{d} \log L_{\mathrm{x}}}=\frac{\mathrm{d} \Phi\left(L_{\mathrm{x}} / e_{1}(z), 0\right)}{\mathrm{d} \log L_{\mathrm{x}}}$,

with the luminosity evolution factor:

$e_{1}(z)=\left\{\begin{array}{ll}(1+z)^{p 1} & \left(z \leq z_{\mathrm{c}}\right) \\ e\left(z_{\mathrm{c}}\right)\left[(1+z) /\left(1+z_{\mathrm{c}}\right)\right]^{p 2} & \left(z>z_{\mathrm{c}}\right)\end{array}\right.$.

We again used the smoothed two power law form (Eq. (2), excluding the $z$-dependent factor) for the $z=0$ SXLF. The best-fit PLE parameters are shown in Table 5 with the results of the $\mathrm{K}-\mathrm{S}$ tests. The best-fit PLE model is overplotted with the binned SXLF in Figs. 4 and 5 as dotted lines. It is apparent from the comparison in these figures, especially the latter, PLE does not represent the behaviour of the low-luminosity $\left(\log L_{\mathrm{x}} \lesssim 44\right)$, intermediate redshift $(0.5 \lesssim z \lesssim 1.8)$ regime, due to the rather restrictive nature of the PLE form.

As a more general analytical form for a refined representation of the SXLF, we have explored the luminositydependent density evolution form (LDDE) form, originally 


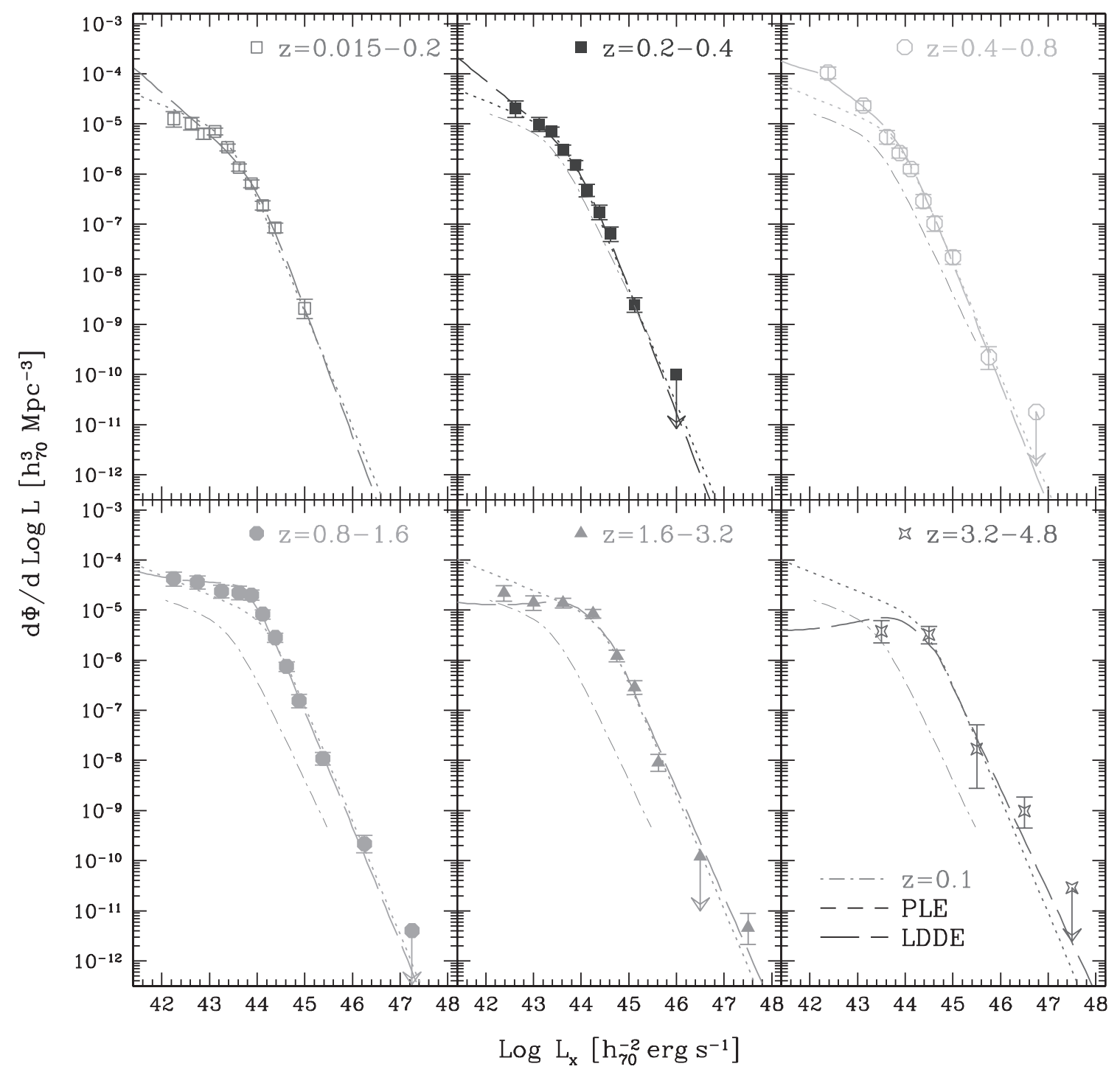

Fig. 4. The soft X-ray luminosity function of the type-1 AGN sample in different redshift shells for the nominal case as labeled. The error bars correspond to $68 \%$ Poisson errors of the number of AGNs in the bin. The best-fit two power law model for the $0.015<z<0.2$ shell are overplotted in the higher redshift panels for reference. The dotted and long-dashed lines give the best-fit PLE and LDDE models discussed in Sect. 4.4 respectively.

suggested by Schmidt \& Green (1983) for describing opticallyselected QSOs:

$\frac{\mathrm{d} \Phi\left(L_{\mathrm{x}}, z\right)}{\mathrm{d} \log L_{\mathrm{x}}}=\frac{\mathrm{d} \Phi\left(L_{\mathrm{x}}, 0\right)}{\mathrm{d} \log L_{\mathrm{x}}} \cdot e_{\mathrm{d}}\left(z, L_{\mathrm{x}}\right)$,

where $e_{\mathrm{d}}\left(z, L_{\mathrm{x}}\right)$ is the density function normalized to $z=0$. The results from Sect. 4.3 show that the peak number density shifts from $z \sim 0.7$ at $\log L_{\mathrm{x}} \sim 42.5$ to $z>2$ at high luminosities. Based on a similar observation of a hard X-ray-selected sample, Ueda et al. (2003) used an expression where $z_{\mathrm{c}}$ is a simple function of $L_{\mathrm{x}}$ :

$e_{\mathrm{d}}\left(z, L_{\mathrm{x}}\right)= \begin{cases}(1+z)^{p 1} & \left(z \leq z_{\mathrm{c}}\right) \\ e_{\mathrm{d}}\left(z_{\mathrm{c}}\right)\left[(1+z) /\left(1+z_{\mathrm{c}}\right)\right]^{p 2} & \left(z>z_{\mathrm{c}}\right)\end{cases}$

along with

$z_{\mathrm{c}}\left(L_{\mathrm{x}}\right)=\left\{\begin{array}{ll}z_{\mathrm{c}, 0}\left(L_{\mathrm{x}} / L_{\mathrm{x}, \mathrm{c}}\right)^{\alpha} & \left(L_{\mathrm{x}} \leq L_{\mathrm{x}, \mathrm{c}}\right) \\ z_{\mathrm{c}, 0} & \left(L_{\mathrm{x}}>L_{\mathrm{x}, \mathrm{c}}\right)\end{array}\right.$.
We use $z_{\mathrm{c}, 44} \equiv z_{\mathrm{c}}\left(L_{\mathrm{x}}=10^{44} \mathrm{erg} \mathrm{s}^{-1}\right)$ as a model parameter. The results of the analysis in the previous section shown in Table 4 suggest that considering the dependence of $p 1$ and $p 2$ on luminosity would still improve the fit. Thus we have also included the following for our full LDDE expression:

$$
\begin{aligned}
& p 1\left(L_{\mathrm{X}}\right)=p 1_{44}+\beta_{1}\left(\log L_{\mathrm{x}}-44\right) \\
& p 2\left(L_{\mathrm{x}}\right)=p 2_{44}+\beta_{2}\left(\log L_{\mathrm{x}}-44\right) .
\end{aligned}
$$

The best-fit parameters and the results of the K-S tests for the PLE and LDDE models are summarized in Table 5. The best-fit PLE and LDDE models are overplotted in Figs. 4 and 5 with dotted and dashed lines respectively. A detailed discussion of the comparison of model and data is given in Sect. 6 .

\section{An alternate approach using the $V_{\max }$ method}

As described in the Introduction, the luminosity function derived from survey data binned in luminosity and redshift does 

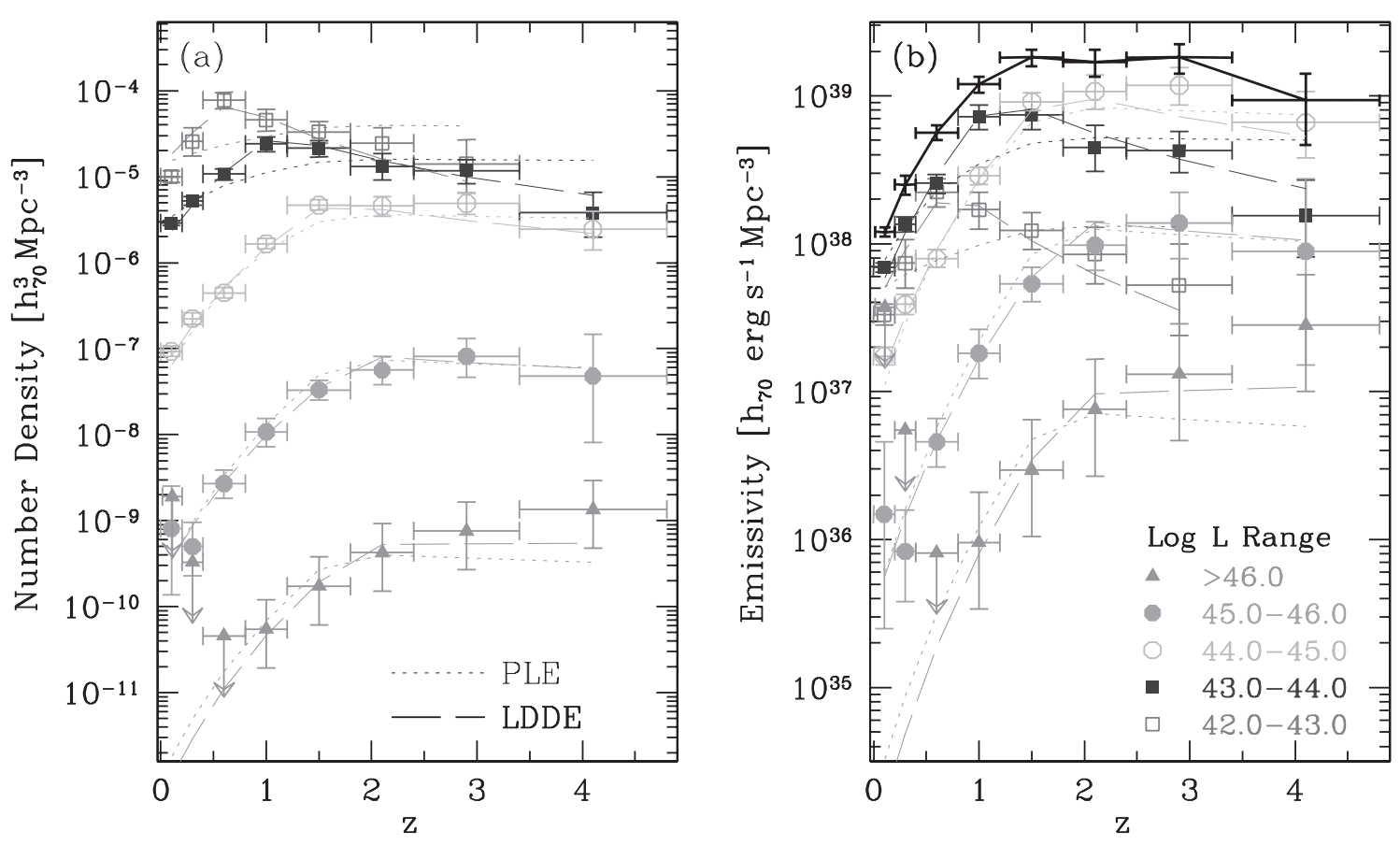

Fig. 5. a) The space density of AGNs as a function of redshift in different luminosity classes and the sum over all luminosities with log $L_{\mathrm{x}} \geq 42$. Densities from the PLE and LDDE models (Sect. 4.4) are overplotted with solid lines. b) The same as a), except that the soft X-ray emissivities are plotted instead of number densities. The uppermost curve (black) shows the sum of emissivities in all luminosity classes plotted.

Table 4. Best-fit evolution parameters for each luminosity bin.

\begin{tabular}{cccccccc}
\hline \hline $\log L_{\mathrm{x}}$-range & $\log L_{\mathrm{xc}}$ & $N$ & $A_{0}$ & $p_{1}$ & $z_{\mathrm{c}}$ & $p_{2}$ & KS-prob $^{b}$ \\
\hline $42.0-43.0$ & 42.5 & 117 & $(7.67 \pm 1.28) \times 10^{-6}$ & $4.90_{-1.12}^{+1.21}$ & $0.65_{-0.12}^{+0.12}$ & $-2.4_{-1.1}^{+1.0}$ & $0.47,0.77,0.64$ \\
$43.0-44.0$ & 43.5 & 381 & $(1.59 \pm 0.15) \times 10^{-6}$ & $3.89_{-0.50}^{+0.43}$ & $1.11_{-0.11}^{+0.22}$ & $-1.8_{-1.1}^{+0.7}$ & $0.55,0.25,0.39$ \\
$44.0-45.0$ & 44.5 & 303 & $(1.83 \pm 0.19) \times 10^{-8}$ & $5.51_{-0.37}^{+0.38}$ & $1.78_{-0.16}^{+0.14}$ & $-1.8_{-1.4}^{+1.3}$ & $0.05,0.47,0.07$ \\
$45.0-46.0$ & 45.5 & 53 & $(4.90 \pm 1.21) \times 10^{-11}$ & $6.06_{-1.22}^{+1.18}$ & $1.79_{-0.26}^{+0.59}$ & $-0.4\left(^{*}\right)$ & $0.81,0.98,0.62$ \\
\hline
\end{tabular}

Parameter values which have been fixed during the fit are labelled by $(*) .{ }^{a}$ Units $-A_{0}: h_{70}^{3} \mathrm{Mpc}^{-3}, L_{\mathrm{x}, *}: 10^{44} h_{70}^{-2} \mathrm{erg} \mathrm{s}^{-1} .{ }^{b} \mathrm{The}$ three values are probabilities in two 1D-KS test for the distribution, $L_{\mathrm{x}}, 1 \mathrm{D}-\mathrm{KS}$ test for the $z$ distribution and the 2D-KS test for the $\left(L_{\mathrm{x}}, z\right)$ space respectively.

not necessarily apply to the centers of the $\left(L_{\mathrm{x}}, z\right)$ bins. This binning bias tends to be especially a problem if data are scarce (often at higher redshifts) and gradients across bins are large. The previous section describes a procedure that corrects the binned space densities to first order.

In this section, we avoid deriving densities from binned survey data. Instead, we use the $V_{\max }$ values of individual RBS sources to derive the zero redshift luminosity function. We then derive by iteration an analytical density template at various $L_{\mathrm{x}}$ values that, together with the zero redshift luminosity function, accounts for the observed counts and redshifts of the deeper surveys. The end result of the procedure is a set of observed values of the luminosity function that apply to the centers of the $\left(L_{\mathrm{x}}, z\right)$ bins, and that is quite insensitive to the precise template employed. A further advantage of employing $V_{\max }$ of individual sources is that it can be derived for two or more selection variables. This allows us to account for the effect of a spectroscopic magnitude limit in some of the deeper surveys beyond which the redshift is unknown for most of the sources. In the first use of $V_{\max }$, this feature was used to derive the luminosity function of radio quasars from a sample in which only the optically brightest objects had redshifts (Schmidt 1968).

\subsection{Using $V_{\max }$ to derive the luminosity function}

The derivation of a luminosity function from objects in a well defined sample usually involves binning the observations in redshift and luminosity. If we make the bins in luminosity so small that each contains only one or zero objects then the luminosity function is composed of contributions from each of the individual sample objects. In the limit, each of the objects contributes to the luminosity function a delta function of amplitude $1 / V_{\max }$ at the object's luminosity $L$, where $V_{\max }$ is the co-moving, density-weighted volume over which the object can be observed within the sample limits in flux and solid angle. This luminosity function will reproduce the source counts of the input sample exactly.

We write the luminosity function as

$\Phi\left(L_{\mathrm{x}}, z\right)=\Phi\left(L_{\mathrm{x}}, 0\right) \rho\left(z, L_{\mathrm{x}}\right)$ 
Table 5. Best-fit parameters for global expressions.

\begin{tabular}{|c|c|c|}
\hline & PLE & LDDE \\
\hline \multicolumn{3}{|c|}{$z=0$ SXLF Parameters } \\
\hline$A_{44}$ & $1.85 \pm .11 \times 10^{-7}$ & $2.62 \pm .16 \times 10^{-7}$ \\
\hline $\log L_{\mathrm{x}, *}$ & $43.33 \pm .09$ & $43.94 \pm .11$ \\
\hline$\gamma_{1}$ & $0.39 \pm .09$ & $0.87 \pm .10$ \\
\hline$\gamma_{2}$ & $2.29 \pm .09$ & $2.57 \pm .16$ \\
\hline \multicolumn{3}{|c|}{ Evolution Parameters } \\
\hline$p 1, p 1_{44}$ & $2.67 \pm .15$ & $4.7 \pm .3$ \\
\hline$z_{\mathrm{c}}, z_{\mathrm{c}, 44}$ & $1.70(+.20 ;-.24)$ & $1.42 \pm .11$ \\
\hline$p 2, p 2_{44}$ & $-0.2 \pm .7$ & $-1.5 \pm .7$ \\
\hline$\alpha$ & $\ldots$ & $0.21 \pm 0.04$ \\
\hline $\log L_{\mathrm{x}, \mathrm{c}}$ & $\ldots$ & $44.67 *$ \\
\hline$\beta_{1}$ & $\ldots$ & $0.7 \pm 0.3$ \\
\hline$\beta_{2}$ & $\ldots$ & $0.6 \pm 0.8$ \\
\hline \multicolumn{3}{|c|}{ K-S Probabilities $\left(L_{\mathrm{x}}, z, 2 D\right)$} \\
\hline $0.015<z<4.8,10$ & $0.10,0.10,0.05$ & $0.86,0.65,0.36$ \\
\hline
\end{tabular}

Parameter values which have been fixed during the fit are labeled by $(*) .{ }^{a}$ Units $-\mathrm{A}: h_{70}^{3} \mathrm{Mpc}^{-3}, L_{\mathrm{x}, *}: 10^{44} h_{70}^{-2} \mathrm{erg} \mathrm{s}^{-1} \cdot{ }^{b}$ The three values are probabilities in two 1D-KS test for the distribution, $L_{\mathrm{x}}, 1 \mathrm{D}-\mathrm{KS}$ test for the $z$ distribution and the 2D-KS test for the $\left(L_{\mathrm{x}}, z\right)$ space respectively.

where $\rho\left(z, L_{\mathrm{x}}\right)$ is the space density, or density evolution, normalized to $z=0$. We approximate $\rho\left(z, L_{\mathrm{x}}\right)$ by an analytical density template. For the redshift dependence we use at low redshifts a power law of $(1+z)$; at higher redshifts we adopt the shape of the density function used by Schmidt et al. (1995) for optically selected quasars:

$$
\begin{array}{ll}
\rho_{\text {tem }}(z)=(1+z)^{m} & 0<z<z_{\mathrm{c}} \\
\rho_{\text {tem }}(z)=\left(1+z_{\mathrm{c}}\right)^{m} & z_{\mathrm{c}}<z<z_{\mathrm{d}} \\
\rho_{\text {tem }}(z)=\left(1+z_{\mathrm{c}}\right)^{m} 10^{k\left(z-z_{\mathrm{d}}\right)} & z>z_{\mathrm{d}} .
\end{array}
$$

We use the RBS which contains 205 AGN-1 in the $\log L_{\mathrm{x}}$ range $42-46$, mostly at low redshifts, to derive the zero redshift luminosity function $\Phi\left(L_{\mathrm{x}}, 0\right)$. The main excercise then is to derive the values of the template parameters $\left(m, z_{\mathrm{c}}, z_{\mathrm{d}}, k\right)$ by fitting to the flux and redshift distributions in the deeper X-ray surveys. This will allow the direct derivation of the luminosity function at the center of bins of luminosity and redshift, as outlined in the steps below.

1. Start the iteration by assuming initial values of the template parameters $\left(m, z_{\mathrm{c}}, z_{\mathrm{d}}, k\right)$ as a function of $L_{\mathrm{x}}$;

2. the zero redshift luminosity function is the sum of delta functions for the assembly of RBS sources

$$
\Phi\left(L_{\mathrm{x}}, 0\right)=\sum_{i=1}^{205}\left(1 / V_{\max , i}^{\mathrm{RBS}}\right) \delta\left(L_{\mathrm{i}}-L_{\mathrm{x}}\right)
$$

where $V_{\max , i}^{\mathrm{RBS}}$ is the accessible density-weighted volume of RBS source $i$ in the RBS, based on its solid angle and flux limit distribution;

3. next, predict expected numbers and redshifts in the deeper surveys. For the part of the luminosity function based on
RBS source $i$, the expected number in survey sur based on the density template is

$n_{\mathrm{tem}}\left(L_{\mathrm{i}}, z\right) \Delta z=\left(V_{\max , i}^{\text {sur }}(z) / V_{\max , i}^{\mathrm{RBS}}\right) \Delta z$

where $V_{\max , i}^{\text {sur }}(z)$ is the accessible density-weighted volume of RBS source $i$ over the redshift range $z, z+\Delta z$ based on the solid angle and flux limit distributions in survey sur;

4. in order to compare with the observed numbers, we use four luminosity classes with $\Delta \log L_{\mathrm{x}}=1.0$ centered on $\log L_{\mathrm{x}}=42.5,43.5,44.5,45.5$ and redshift shells of $\Delta \log z=0.1$ centered on $\log z=-0.95,-0.85, \ldots, 0.75$. Total binned numbers predicted in all the non-RBS surveys are $N_{\text {tem }}\left(L_{\mathrm{x}}, z\right)$;

5. next, use the observed number $N_{\text {obs }}\left(L_{\mathrm{x}}, z\right)$ in each $\left(L_{\mathrm{x}}, z\right)$ bin to derive the space density or luminosity function at the center of each luminosity bin by scaling the template value of the luminosity function:

$\Phi_{\text {obs }}\left(L_{\mathrm{x}}, z\right)=\Phi\left(L_{\mathrm{x}}, 0\right) \rho_{\mathrm{tem}}\left(z, L_{\mathrm{x}}\right) \cdot \frac{N_{\mathrm{obs}}\left(L_{\mathrm{x}}, z\right)}{N_{\mathrm{tem}}\left(L_{\mathrm{x}}, z\right)}$

6. comparison of $\Phi_{\mathrm{obs}}\left(z, L_{\mathrm{x}}\right)$ and $\Phi\left(L_{\mathrm{x}}, 0\right) \rho_{\mathrm{tem}}\left(z, L_{\mathrm{x}}\right)$ serves as a guide for the next iteration of the template parameters $\left(m, z_{\mathrm{c}}, z_{\mathrm{d}}, k\right)$, starting at step 2 above.

\subsection{Deriving $V_{\max }$ values}

The values of $V_{\max , i}^{\text {sur }}$ are based on the maximum accessible redshifts $z_{\max , i}^{\text {sur }}$ of RBS source $i$ in survey sur. For the SA-N, NEPS, RIXOS, RMS and CDF-S surveys, where missing redshifts are not correlated with X-ray or optical flux, we assume that $z_{\max , i}^{\text {sur }}$ equals the $\mathrm{X}$-ray redshift limit $z_{\mathrm{lim}, \mathrm{x}}$. In accounting for the missing redshifts, we assume that their distribution is similar to that of the observed redshifts and use an effective solid angle appropriately reduced from the geometric solid angle. 


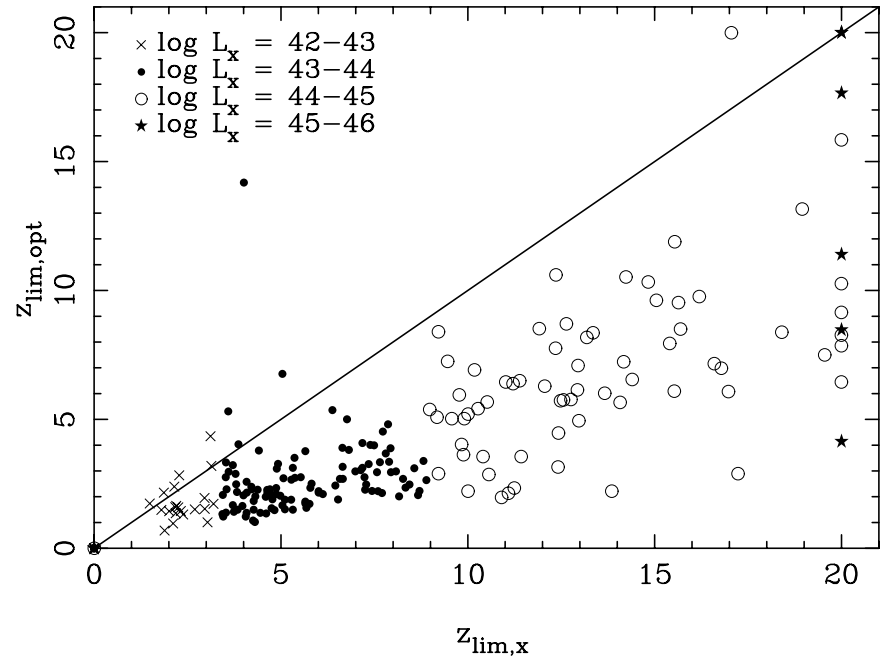

Fig. 6. The X-ray redshift limit versus the optical redshift limit for 205 RBS AGN-1 sources. In this illustration the X-ray flux limit is $10^{-16} \mathrm{cgs}$ and the magnitude limit $R=24$, similar to the case of the CDF-N.

For the RDS/XMM and CDF-N surveys, where redshifts are missing primarily for optically very faint objects, we proceed as follows. In this case, we employ only objects brighter than a magnitude limit $m_{\text {lim }}$ (in practice $R<24$ ) and use the full geometric survey solid angle. In predicting the number of expected objects in survey sur, this introduces a second limiting redshift, $z_{\text {lim,opt }, i}$ that will depend on the optical luminosity of RBS source $i$ contributing to the luminosity function. The relevant limit $z_{\max , i}^{\text {sur }}$ for the derivation of the expected counts is the smaller of $z_{\text {lim,x }, i}$ and $z_{\text {lim,opt }, i}$.

The determination of $z_{\text {lim,opt }, i}$ is based on the magnitudes of the RBS objects. Among the 205 RBS AGN-1, Salvato (2002) has carried out optical photometry of a redshift-limited sample of 89 sources, deriving magnitudes for the nucleus, disk and spheroid. Comparison of the total magnitudes given by Salvato with the RBS magnitudes shows $\left\langle R_{\mathrm{RBS}}-R_{\mathrm{Sal}}\right\rangle=$ -0.5 with a dispersion of 0.9 mag. We apply this correction to the 116 remaining RBS sources. Based on the systematics of the Salvato magnitudes, we find that on the average, the absolute $R$-magnitude of the galaxy light is -21.3 , that the disk light accounts for $56 \%$ of the galaxy light, and that for low-luminosity objects the nucleus contributes no less that $25 \%$ of the total light. This allows the, admittedly uncertain, derivation of the nuclear, disk and spheroid magnitudes of the remaining 116 RBS objects. We use the following values for the spectral index $\alpha=\mathrm{d} \log S / \mathrm{d} \log v$ of the nuclear, disk and spheroid components, estimated from spectral energy distributions given by Kinney (1996): $\alpha_{\text {nuc }}=-0.5$, $\alpha_{\text {dis }}=-1.0, \alpha_{\text {sph }}=-3.0$. These values allow evaluation of the $\mathrm{K}$-correction required to derive the redshift $z_{\text {lim,opt }, i}$ at which each of the 205 RBS sources reaches the spectroscopic magnitude limit $R_{\text {lim }}=24$. We illustrate in Fig. 6 the effect of the optical limit on the redshift limits for the CDF-N: for objects below the diagonal line, the redshift limit is that set by the optical limit.

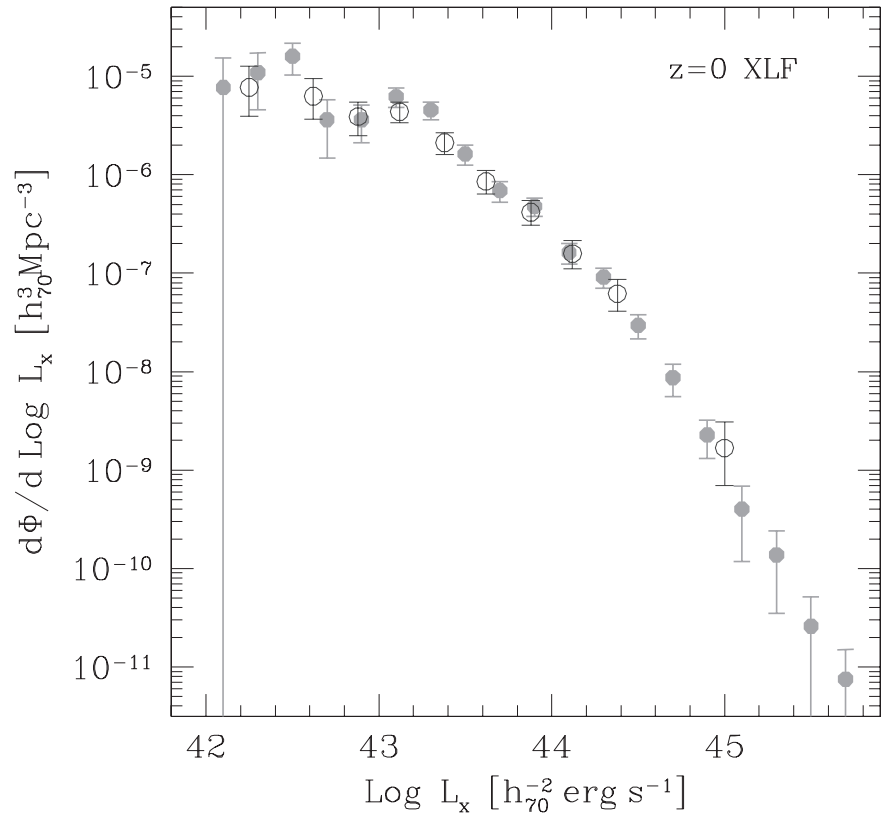

Fig. 7. The $z=0$ luminosity function $\Phi\left(L_{\mathrm{x}}, 0\right)$ derived from 205 RBS AGN-1 sources using the $V_{\max }$ method (red filled symbols), compared with the binned estimate in the redshift shell $z=0.015-0.2$, corrected to $z=0$.

\subsection{The zero redshift $X$-ray luminosity function}

The zero redshift luminosity function $\Phi\left(L_{\mathrm{X}}, 0\right)$ is derived from the RBS sources as outlined in Sect. 5.1, Eq. (17). Since the accessible volume $V_{\max , i}^{\mathrm{RBS}}$ is density-weighted, this derivation requires information about the density template, discussed in the next section. At the low redshifts of the RBS objects (typically 0.1 ), this involves only the $m$ parameter of the low redshift $(1+z)^{m}$ density variation. A binned version of $\Phi\left(L_{\mathrm{x}}, 0\right)$, created by summing the delta functions in bins of $\Delta \log L_{\mathrm{x}}=$ 0.2 , is shown in Fig. 7.

\subsection{The analytical density template $\rho\left(z, L_{\mathrm{x}}\right)$}

The density template defined in Eqs. (15) and (16) with parameters $\left(m, z_{\mathrm{c}}, z_{\mathrm{d}}, k\right)$ is an analytical approximation of the density function $\rho\left(z, L_{\mathrm{x}}\right)$. The derivation of the template parameters was carried out as follows. As described in Sect. 5.1, it is a procedure of trial and error. For each of the four luminosity classes, in iterating the value of $m$, we minimize $\left\langle N_{\text {obs }}\left(L_{\mathrm{x}}, z\right)-N_{\text {tem }}\left(L_{\mathrm{x}}, z\right)\right\rangle$ for $0.5<z<z_{\mathrm{c}}$, since at low redshifts the luminosity function is firmly anchored by the RBS objects. The values of $z_{\mathrm{c}}, z_{\mathrm{d}}$ and $k$ are derived by minimizing $\left\langle N_{\text {obs }}\left(L_{\mathrm{x}}, z\right)-N_{\text {tem }}\left(L_{\mathrm{x}}, z\right)\right\rangle$ for $z>z_{\mathrm{c}}$, by adjusting $k$ for the lower luminosity classes to fit the total observed AGN-1 in the CDF-S and CDF-N (regardless of availability of redshifts or identification), and for the higher luminosity classes by adopting parameter values for optically selected quasars (see next section). This procedure makes it difficult to evaluate the statistical significance of the templates. We will show below that the accuracy of the templates actually has a negligible effect on the values of $\Phi_{\mathrm{obs}}\left(L_{\mathrm{x}}, z\right)$. 

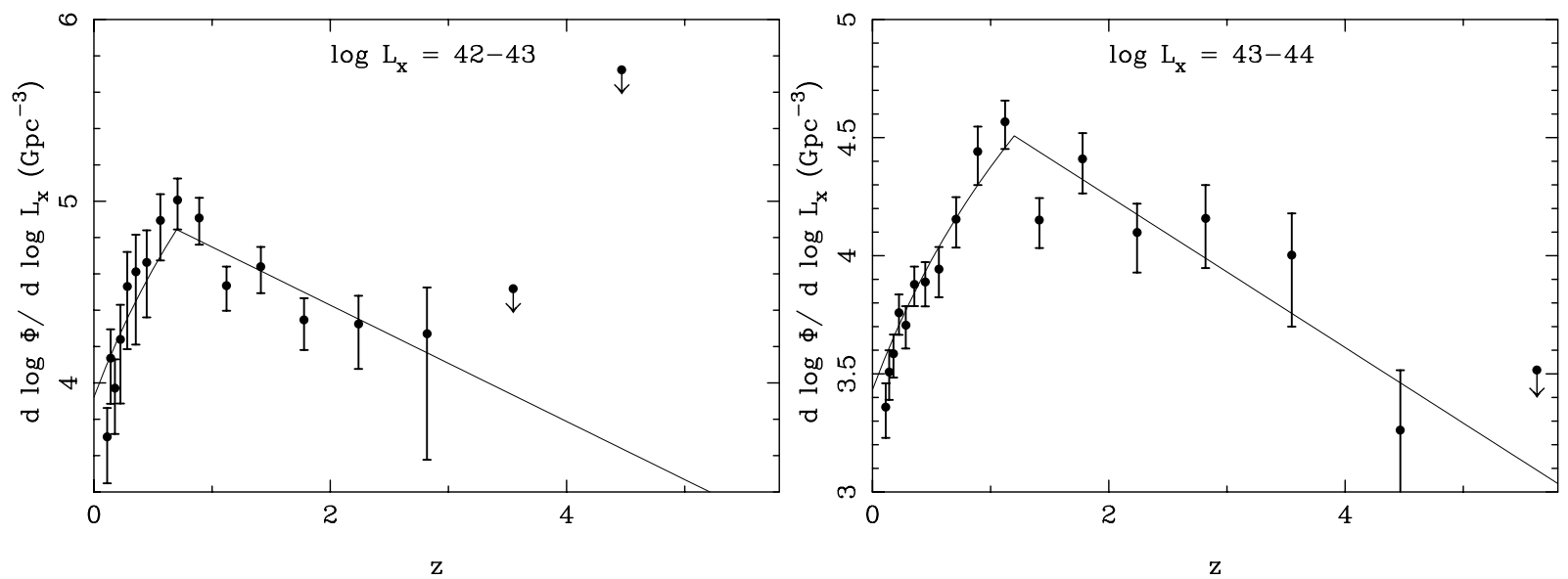

Fig. 8. The luminosity function as a function of redshift $z$. The $z=0$ values are based on 21 RBS sources for $\log L_{\mathrm{x}}=42-43,111$ sources for $\log L_{\mathrm{x}}=43-44$. The points are based on numbers observed in the non-RBS surveys. The thin line represents the density template (see text for details).
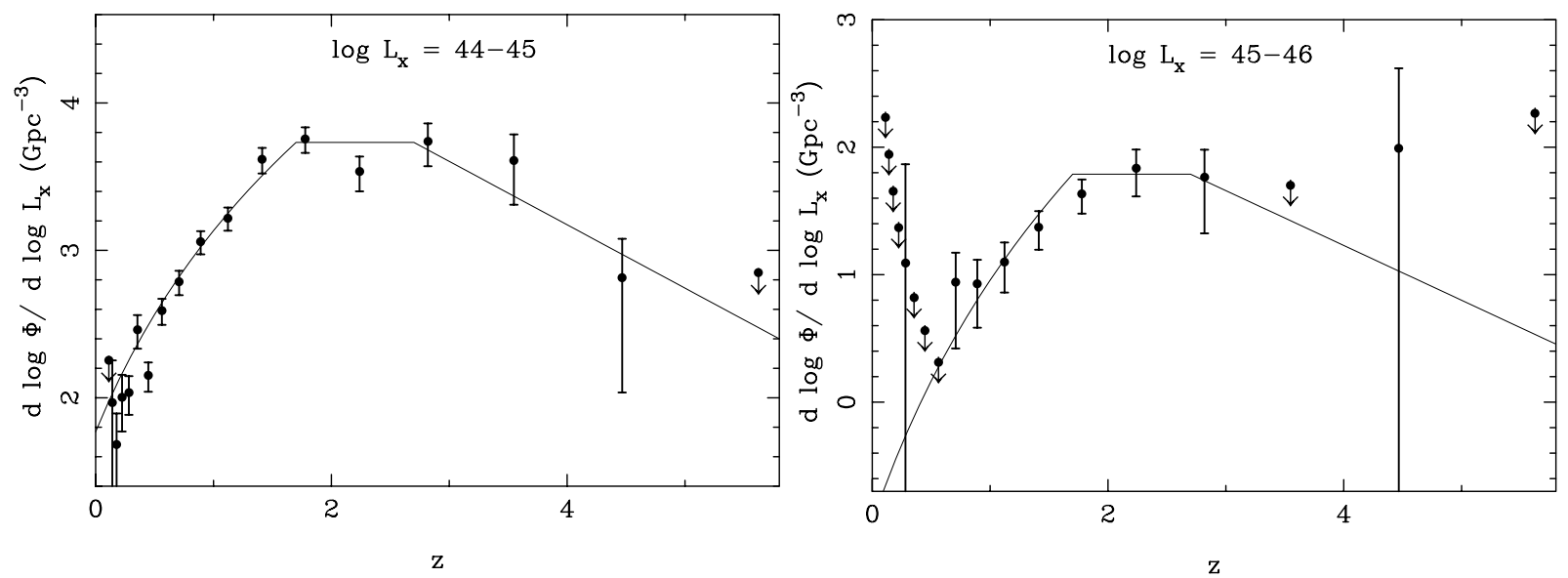

Fig. 9. The luminosity function as a function of redshift $z$. The $z=0$ value are based on 67 RBS sources for $\log L_{x}=44-45,6$ sources for $\log L_{\mathrm{x}}=45-46$. The points are based on numbers observed in the non-RBS surveys. The thin line represents the density template; the parameters of the template for $z>1.7$ were adopted from those found for high-luminosity optical quasars (see text for details).

Table 6. Parameters characterizing the density template.

\begin{tabular}{lcccc}
\hline \hline $\log L_{\mathrm{x}}$ & 42.5 & 43.5 & 44.5 & 45.5 \\
\hline$m$ & $4.0 \pm 0.7$ & $3.4 \pm 0.5$ & $5.0 \pm 0.2$ & $7.1 \pm 1.0$ \\
$z_{\mathrm{c}}$ & 0.7 & 1.2 & 1.7 & 1.7 \\
$z_{\mathrm{d}}$ & 0.7 & 1.2 & 2.7 & 2.7 \\
$k$ & -0.32 & -0.32 & -0.43 & -0.43 \\
\hline
\end{tabular}

The resulting values of $\left(m, z_{\mathrm{c}}, z_{\mathrm{d}}, k\right)$ for the four luminosity classes are given in Table 6 . The errors for the low-redshift power index $m=\mathrm{d} \log \rho / \mathrm{d} \log (1+z)$ have been estimated using the number of observed objects in the redshift shells $0.5<z<$ $z_{\mathrm{c}}$ in comparison with the RBS objects. From this analysis and the results shown in Table 4 it appears that $m$ exhibits a significant increase with $L_{\mathrm{x}}$ for $\log L_{\mathrm{x}}>43.0$. For these luminosities, we employ a quadratic interpolation of $m$ with $\log L_{\mathrm{x}}$ through the values given in the table. For $\log L_{\mathrm{x}}=42-43$ we assume $m$ to be constant.

\subsection{The $X$-ray luminosity function $\Phi_{\mathrm{obs}}\left(L_{\mathrm{x}}, z\right)$}

The observed values of the luminosity function $\Phi_{\mathrm{obs}}\left(L_{\mathrm{x}}, z\right)$ are obtained by scaling the template luminosity function by a factor $N_{\text {obs }}\left(L_{\mathrm{x}}, z\right) / N_{\text {tem }}\left(L_{\mathrm{x}}, z\right)$, see Eq. (19). These values of $\Phi_{\text {obs }}\left(L_{\mathrm{x}}, z\right)$ are plotted versus the redshift for each of the four luminosity classes in Figs. 8 and 9. The $\pm 1 \sigma$ error bars are based on the numbers $N_{\text {tem }}\left(L_{\mathrm{x}}, z\right)$ predicted by the template. The figures also show the template luminosity function resulting from the product of the zero redshift luminosity function $\Phi\left(L_{\mathrm{x}}, 0\right)$ and the template density function $\rho_{\text {tem }}\left(z, L_{\mathrm{x}}\right)$.

The $\pm 1 \sigma$ error bars do not include any contribution reflecting the error of the template density function $\rho_{\text {tem }}\left(z, L_{\mathrm{x}}\right)$. We explore the effect of the template by rederiving the predicted number $N_{\text {tem }}\left(L_{\mathrm{x}}, z\right)$ on the extreme assumption that $m=0$ and $k=0$, i.e. that there is zero density evolution. We find that the observed densities $\log \Phi_{\text {obs }}\left(L_{\mathrm{x}}, z\right)$ increase by only $0.00-0.06$. Since the template errors must be much smaller than assumed in this extreme example, their effect on the error of $\Phi_{\mathrm{obs}}\left(L_{\mathrm{x}}, z\right)$ is negligible. The main result of this section is the set of $\Phi_{\mathrm{obs}}\left(L_{\mathrm{x}}, z\right)$ values plotted in Figs. 8 and 9; the 
templates serve primarily to eliminate the uncertainty related to binning.

For $\log L_{\mathrm{x}}=42-43$ and $43-44$, the density rises by an order of magnitude to $z \sim 0.7$ and 1.2 , respectively, and then declines steadily (Fig. 8). As shown in the next section, the peak at $z \sim 0.7$ for $\log L_{\mathrm{x}}=42-43$ is little affected by redshift spikes caused by large-scale structure.

The density distribution for AGN-1 with $\log L_{\mathrm{x}}=44-45$ is documented to $z \sim 4$ (Fig. 9). Since in this case, the evidence for a decline in density at high redshift was not initially clear, we adopted for the parameters $\left(z_{\mathrm{c}}, z_{\mathrm{d}}, k\right)$ of the template above $z=1.7$ those found for high-luminosity optical quasars by Schmidt et al. (1995). It appears from Fig. 9 that the X-ray data are consistent with the adopted shape. In order to further investigate whether the space density declines significantly at high redshift, we explore a test template in which the density does not decline at all, i.e. remains flat at high redshifts $(k=0)$. The results are shown in Fig. 10. The error bars are much reduced from those in Fig. 9, reflecting the fact that our errors are based on the predicted numbers, which are larger for a flat evolution function at high redshift. The bins at $z=4.47,5.62,7.07$ have observed/predicted numbers of $1 / 7.9,0 / 7.2$, and $0 / 19.3$, respectively. Limiting the case to $z<6.3$, for which the observation of redshifts in high-luminosity quasars should be no problem, we have for $z>4$ one observed object versus 15 expected. The Poisson probability for such an occurence barring systematic effects is $3 \times 10^{-7}$, constituting strong evidence that for $\log L_{\mathrm{x}}=44-45$ the space density declines beyond $z \sim 4$.

The low-redshift density parameter $m$ for $\log L_{\mathrm{x}}=45-46$ continues the trend of an increasing $m$ for larger $L_{\mathrm{x}}$. At large redshift, the sparse data do not give any information about the density beyond $z \sim 3$ (see Fig. 9).

\section{Discussion}

In the present paper we have used two different methods to derive the AGN-1 X-ray luminosity function and its evolution. Detailed descriptions of the two methods are given in Sects. 4.1 and 5.1. Conceptually, the binned method derives a first order luminosity function by dividing the numbers $N_{\text {obs }}\left(L_{\mathrm{x}}, z\right)$ observed in the input surveys by the appropriate volumes. An analytical representation of the luminosity function is used to predict the numbers $N_{\mathrm{mdl}}\left(L_{\mathrm{x}}, z\right)$ expected in these surveys. The luminosity function is then corrected by the factor $N_{\mathrm{obs}} / N_{\mathrm{mdl}}$.

In the $V_{\max }$ method, the RBS is used to derive the zero redshift luminosity function. An analytical density template is used to predict the numbers $N_{\text {tem }}\left(L_{\mathrm{x}}, z\right)$ expected in the deeper surveys. In this process, the effect of a spectroscopic magnitude limit on the $V_{\max }$ values is included for deep surveys where this limit applies. Once the template predictions are close to the observed numbers $N_{\text {obs }}\left(L_{\mathrm{x}}, z\right)$ in the deeper surveys, the luminosity function at the center of each $\left(L_{\mathrm{x}}, z\right)$ bin is derived by multiplying the template luminosity function by $N_{\text {obs }} / N_{\text {tem }}$.

Using the optical magnitudes and spectra of the RBS sources in deriving the redshifts at which they would reach the spectroscopic limit $R=24$ introduces uncertainties. The magnitudes of the 116 RBS sources not studied by Salvato (2002) are quite poor and so is their separation in nucleus, disk

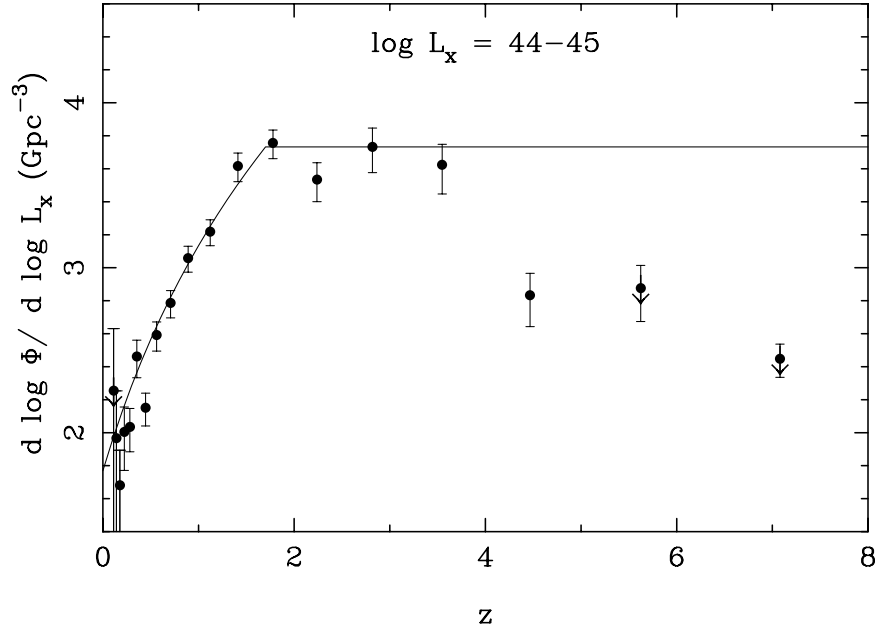

Fig. 10. The luminosity function versus redshift $z$, derived using a template having constant space density at large redshift. The observed numbers at $z=4.47,5.62,7.08$ are $1,0,0$, respectively. This plot gives clear evidence that high-luminosity X-ray AGNs type 1 show a decline in space density beyond $z \sim 4$.

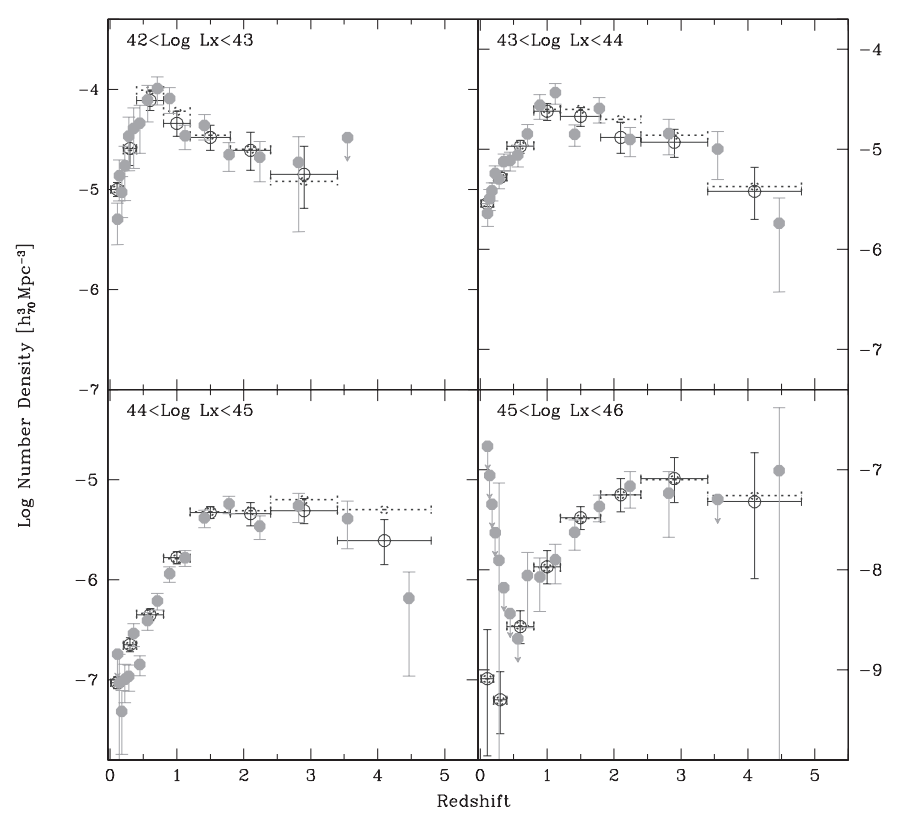

Fig. 11. Comparison between the space densities derived with two different methods. The blue datapoints with error bars refer to the binned treatment using the $N_{\mathrm{obs}} / N_{\text {mdl }}$ method, the dashed horizontal lines corresponding to the maximum contribution of unidentified sources. The red dots refer to the $V_{\max }$ method and the data given in Figs. 8 and 9.

and spheroid components. The assumed spectra of these components are schematic. Obtaining spectral energy distributions for all the RBS sources down to the far UV would allow deriving their flux directly at or near the rest wavelengths corresponding to the $R$ magnitude at the limiting redshifts.

It is reassuring that the general properties and absolute values of the space density are very similar in the two different derivations in Sects. 4 and 5. Figure 11 shows a direct comparison between the binned and $V_{\max }$ determinations of the space density, which agree very well within statistical errors. 
We use overall fits to the luminosity function and its cosmological evolution in order to compare to previous work and to enable theoretical calculations with simplified analytical forms. For this purpose, we use two functional forms, i.e. a pure luminosity evolution (PLE) and a luminosity-dependent density evolution (LDDE) model. Assuming PLE, the luminosity evolution index $p 1 \approx 2.7$ and the cutoff redshift $z_{\mathrm{c}} \approx 1.7$ are in rough agreement with previous results from type-1 AGNs in ROSAT or Einstein/ROSAT combined surveys (e.g. Paper I; Jones et al.1997; Page et al. 1997; Boyle et al. 1993).

The results of the K-S test for the PLE fit over the whole sample are marginally acceptable, with $\sim 5 \%$ chance of obtaining a $2 \mathrm{D} \mathrm{K}-\mathrm{S}$ value larger than observed. However, this is caused by the sheer number of AGNs in the part of the $z-L_{\mathrm{x}}$ space where PLE is still a good description, which dominates the overall statistics. Figure 5 clearly shows that PLE fails to reproduce the behavior of the SXLF around $\log L_{\mathrm{x}} \lesssim 44$ at $0.4 \lesssim z \lesssim 1.7$. The luminosity bin $42<\log L_{\mathrm{x}}<43.5$ in the $0.4<z<0.8$ shell contains 41 objects where PLE predicts 19 and the adjacent regime of $43.0<\log L_{\mathrm{x}}<44.0$ at $0.8<z<1.2$ contains 46 objects where PLE predicts 25 , so that the overall excess corresponds to more than $4.5 \sigma$. In constructing the LDDE form, we tried to fully represent the SXLF from our data with unprecedented redshift and luminosity coverage. The overall $2 \mathrm{D} \mathrm{K}-\mathrm{S}$ acceptance of LDDE has been improved to $36 \%$. The only location where the LDDE model still deviates from the data significantly is the very end of our sample, the $42<\log <43$ bin at $0.015<z<0.2$, where LDDE predicts 66 objects while we observed 45 objects (a $\sim 3 \sigma$ deviation).

Even though our sample is a soft X-ray-selected type-1 AGN sample, the overall behaviour of our XLF is similar to that obtained by Ueda et al. (2003) for the intrinsic (de-absorbed) luminosity function of hard X-ray selected obscured and unobscured AGN. To make this comparison, we have refitted our sample with the LDDE model where $\beta_{1}$ and $\beta_{2}$ are fixed to zero. This is exactly the same as the function form which Ueda et al. (2003) used to describe their intrinsic HXLF. All the $z=0$ XLF parameters and evolution parameters are remarkably close between our SXLF and HXLF, except the global normalization. The HXLF normalization is found to be about five times larger than that of our SXLF, after adjustments for the differences in energy bands and the difference in the luminosities at which normalizations are evaluated. This factor probably accounts for the absorbed objects missing in the SXLF. However, the Ueda et al. sample, containing about 250 AGN, is limited to lower luminosities and lower redshifts than our sample of 1000 objects, so that its statistical quality and limited sensitivity range were not sufficient to constrain the decline of the space density at high redshifts, which has been measured significantly in our sample for the first time.

Very recently, Barger et al. (2005) have presented X-ray luminosity function analyses both in the hard and soft X-ray bands, based on the CDF-N, CDF-S, CLASXS and ASCA surveys. Again, their results are in good agreement with the soft XLF discussed here and the hard XLF presented by Ueda et al. however, they still suffer from substantial identification incompleteness. Also, their results on broad-line AGN are not directly

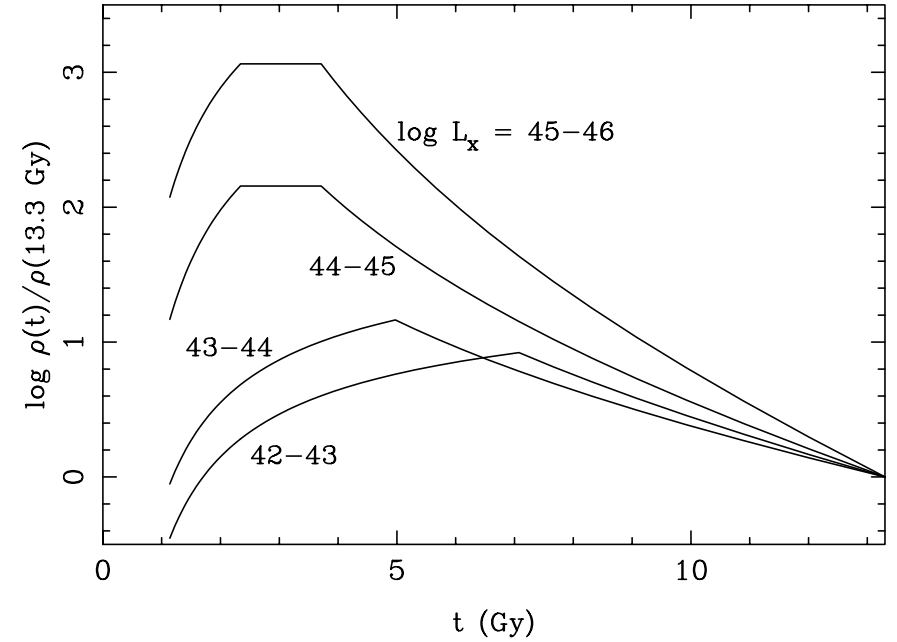

Fig. 12. Co-moving density of AGN-1 versus cosmic time normalized to the present time.

comparable to our type-1 AGN sample, because they only include the optically classified type-1 AGN and thus miss most of the low-luminosity unabsorbed AGN-1 we are concerned with in this paper. A critical comparison of our XLF with those of Ueda et al. (2003) and Barger et al. (2005) will be the topic of a future paper.

The evidence for a peak in the evolution function is quite strong at $z \sim 0.7$ for $L_{\mathrm{x}}=42-43$ (see Fig. 11). The faintest end of the sample depends on the small field of Chandra Deep Field-South and there is some concern on the effects of the large-scale structure and cosmic variance associated with it. In particular, Gilli et al. (2003) found two redshift spikes, one at $z \sim 0.67$ and the other at $z \sim 0.73$. One may wonder whether the $z=0.7$ peak is caused by these redshift spikes (see also Gilli et al. 2003). These do, however, not affect our SXLF estimates significantly. In the analysis in Sect. 4 only six out of 41 AGNs $(15 \%)$ in our sample in the $0.4<z \leq 0.8$ bin in the range $42 \leq \log L_{\mathrm{x}}<43.5$ are in these spikes $(0.664 \leq z \leq 0.685$ and $0.725 \leq z \leq 0.742)$ and this is the only regime where the spikes give a non-negligible contribution. In the analysis in Sect. 5, the $z=0.71$ bin contains 5 objects from the two spikes, for a total of 5 out of 15 observed objects. Disregarding these objects would decrease the derived density by dex 0.22 in Fig. 8, actually leading to better agreement with the template densities. We therefore conclude that cosmic variance is not significantly affecting our results on the evolution of the space density.

We show in Fig. 12 the AGN-1 space density as a function of cosmic time. We see dramatic changes with $L_{\mathrm{x}}$. For declining $L_{\mathrm{x}}$ as we move from high-luminosity AGN or quasars to Seyfert galaxies, the main formation of the objects occurred at later cosmic times. For $\log L_{\mathrm{x}}>44$ the density curve is similar to that for quasars. It is an intriguing question whether the observed dependence of $m$ on $L_{\mathrm{x}}$ is accompanied by a corresponding dependence on $L_{\text {opt }}$. At $\log L_{\mathrm{x}}<44$ the AGN-1 are mostly Seyfert galaxies, for which there is no comparable optical evidence about their density curve. 
These new results paint a dramatically different evolutionary picture for low-luminosity AGN compared to the highluminosity QSOs. Obviously, the rare, high-luminosity objects can form and feed very efficiently rather early in the universe. Their space density declines by more than two orders of magnitude at redshifts below $z=2$. The bulk of the AGN, however, has to wait much longer and shows a decline of space density by less than a factor of 10 at redshifts below one. The late evolution of the low-luminosity Seyfert population is very similar to that which is required to fit the Mid-infrared source counts and background (Franceschini et al. 2002) and also the bulk of the star formation in the Universe (Madau et al. 1998), while the rapid evolution of powerful QSOs traces more the merging history of spheroid formation (Franceschini et al. 1999).

This kind of anti-hierarchical Black Hole growth scenario is not predicted in most of the semi-analytic models based on Cold Dark Matter structure formation models (e.g. Kauffmann \& Haehnelt 2000; Wyithe \& Loeb 2003). This could indicate two modes of accretion and black hole growth with radically different accretion efficiency (see e.g. Duschl \& Strittmatter 2002). A self-consistent model of the black hole growth which can simultaneously explain the anti-hierarchical X-ray space density evolution and the local black hole mass function derived from the $M_{\mathrm{BH}}-\sigma$ relation assuming two radically different modes of accretion has recently been presented by Merloni (2004).

Finally, we compare the space density of soft X-ray selected QSOs from our sample to the one of optically-selected QSOs at the most luminous end. The comparison is plotted in Fig. 13. The $z<2$ number density curve for optically selected QSOs $\left(M_{b_{\mathrm{J}}}<-26.0\right)$ is from the combination of the $2 \mathrm{dF}$ and $6 \mathrm{dF}$ QSO redshift surveys by Croom et al. (2004). The $z>2.7$ number densities from Schmidt et al. (1995) and Fan et al. (2001) have been originally given for $h_{70}=5 / 7, \Omega_{\mathrm{m}}=1, \Omega_{\Lambda}=0$. Their data points have been converted to our default cosmology and the $M_{\mathrm{B}}$ threshold has been re-calculated with an assumed spectral index of $\alpha_{\mathrm{o}}=-0.79$ $\left(f_{v} \propto v^{\alpha_{\circ}}\right)$, following e.g. Vignali et al. (2003). The plotted curve from Schmidt et al. / Fan et al. is for $M_{\mathrm{B}}<-26.47$ under these new assumptions. A small correction of densities due to the cosmology conversion causing redshift-dependent luminosity thresholds has also been made, assuming $\mathrm{d} \Phi / \mathrm{d} \log L_{\mathrm{B}} \propto$ $L_{\mathrm{B}}^{-1.6}$ (Fan et al. 2001). The space density for the soft X-ray QSOs for the luminosity class $44<\log L_{\mathrm{x}}<45$ has been plotted both for the binned and $V_{\max }$ determination. The Croom et al. (2004) space density had to be scaled up by a factor of 16 in order to match the X-ray density at $z \sim 2$. The Schmidt et al. / Fan et al. data points have been scaled by a factor of 40 to match the soft X-ray data at $z=2.7$ in the plot. There is relatively little difference in the density functions between the $\mathrm{X}$-ray and optical QSO samples, although we have to keep in mind, that both the rise and the decline of the space density is varying with X-ray luminosity, so that this comparison can only be illustrative until larger samples of high-redshift X-ray selected QSOs will be available.

Very recently, Wall et al. (2005), have presented an update of the space density evolution of the Parkes quarter-Jansky sample of flat-spectrum radio sources. They basically confirm

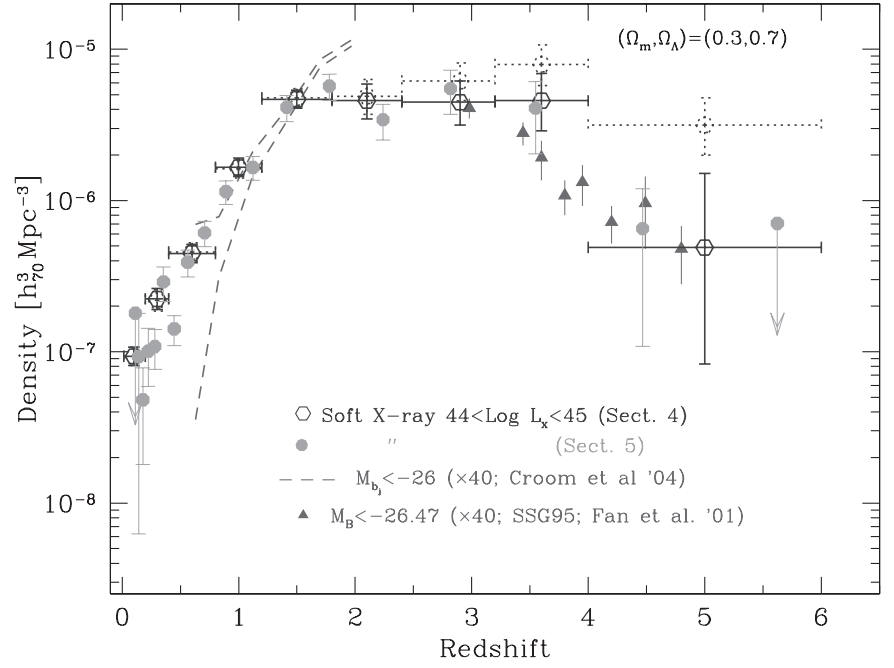

Fig. 13. Comparison of the space density of luminous QSOs between optically selected and soft X-ray selected samples. The X-ray number densities are plotted for the luminosity class $\log L_{\mathrm{x}}=44-45$, both for the binned and $V_{\max }$ analysis with the same symbols as in Fig. 11. The dashed lines in represent the one sigma range for $M_{b_{\mathrm{j}}}<-26.0$ from Croom et al. (2004), multiplied by a factor of 40 to match the $\mathrm{X}$-ray space density at $z=1.5$. The triangles at $z>2.7$ with $1 \sigma$ errors are from Schmidt et al. (1995) (SSG95) and Fan et al. (2001) after a cosmology conversion (see text) and a scaling by a factor of 40 to match with the soft X-ray density at $z \sim 2.7$. As discussed in this paper, both the rise and the decline of the space density change with $L_{\mathrm{x}}$ and therefore this comparison can only be illustrative.

the rise and fall of the QSO population as now seen both in the optical and X-ray QSO populations.

\section{Conclusions and outlook}

We have merged the Chandra and XMM-Newton deep survey data with the whole body of previously identified ROSAT AGN samples. We have selected only the type-1 AGN in all samples and treated only the detections and $\mathrm{X}$-ray fluxes in the $0.5-2 \mathrm{keV}$ band. The different samples cover an unprecedented five orders of magnitude in flux limit and six orders of magnitude in survey solid angle between the ROSAT Bright and serendipitous surveys, the XMM-Newton Lockman Hole survey and the Chandra Deep Surveys. The sample comprises 944 identified AGN-1 objects and only 57 unidentified sources, which could be $\mathrm{AGN}-1$, i.e. roundabout 1000 objects. The luminosity-redshift diagram is almost homegeneously filled with our sample objects. With this sample we arrive at the following conclusions:

1. The new Chandra and XMM-Newton sources are predominantly Seyfert galaxies at a median luminosity of $\sim 10^{43} \mathrm{erg} \mathrm{s}^{-1}$ and a median redshift around 0.7 and push the determination of the X-ray luminosity and space density functions into so far unexplored parameter ranges of redshift and luminosity.

2. AGN-1 are by far the largest contributors to the soft X-ray selected samples. Their evolutional properties are responsible for the break in the total X-ray sources counts in the $0.5-2 \mathrm{keV}$ band. 
3. The soft X-ray luminosity function of AGN shows a clear change of shape as a function of redshift, confirming earlier reports of luminosity-dependent density evolution for optical quasars and X-ray AGN.

4. The space density function changes significantly for different luminosity classes. It shows a strong positive evolution, i.e. a density increase at low redshifts up to a certain redshift and then a flattening. The redshift, at which the evolution peaks, changes considerably with X-ray luminosity, from $z \approx 0.5-0.7$ for luminosities $\log L_{\mathrm{x}}=42-43 \mathrm{erg} \mathrm{s}^{-1}$ to $z \approx 2$ for $\log L_{\mathrm{x}}=45-46 \mathrm{erg} \mathrm{s}^{-1}$.

5. The amount of density evolution from redshift zero to the maximum space density is also a strong function of X-ray luminosity. The change is more than a factor of 100 at high luminosities, similar to what has been observed for optically selected QSOs, but it is less than a factor of 10 for low X-ray luminosities.

6. For the first time, we find a clear decline of the space density of X-ray selected AGN towards high redshift, using a rigorous treatment of optical incompleteness and the corresponding survey volume. The decline is observed clearly for X-ray luminosities in the range $\log L_{\mathrm{x}}=42-45 \mathrm{erg} \mathrm{s}^{-1}$, while at higher luminosities the survey volume at highredshift is still too small to obtain meaningful densities.

In the future, X-ray surveys which are both wide and deep are necessary, in order to provide enough volume for a better measurement of the space density function of the rare high-luminosity AGN at large redshifts. Several new surveys towards this goal are already underway, e.g. the Chandra Multiwavelength Project (Champ) (Silverman et al. 2005), the XMM-Newton AXIS project (Barcons et al. 2002), the Chandra Large Area Synoptic X-Ray Survey (CLASXS) (Yang et al. 2004; Steffen et al. 2004), the Extended Chandra Deep Field South (PI: W.N. Brandt) and the XMM-Newton COSMOS Field (PI: G. Hasinger), which together should enrich the sample of $z>4$ objects by about an order of magnitude. Ultimately, new X-ray Dark Energy missions, aiming to survey large solid angles on the sky to considerable depth could provide a factor of 100-1000 increase in AGN sample size.

Acknowledgements. Part of this work was supported by the German Deutsches Zentrum für Luft- und Raumfahrt, DLR grant number 50 OR 0207 . Also this work is partially supported by NASA grant NAG 5-10875 (LTSA). T.M. appreciates support from Max-Planck Society during his visits to MPE. We thank an anonymous referee for constructive comments, which significantly improved the paper.

\section{References}

Alexander, D. M., Bauer, F. E., Brandt, W. N., et al. 2003, AJ, 126, 539

Appenzeller, I., Thiering, I., Zickgraf, F.-J., et al. 1998, A\&AS, 117, 319

Barcons, X., Carrera, F. J., Watson, M. G., et al. 2002, A\&A, 382, 522 Barger, A. J., Cowie, L. L., Mushotzky, R. F., \& Richards, E. A. 2001, AJ, 121, 662

Barger, A. J., Cowie, L. L., Capak, P., et al. 2003, AJ, 126, 632

Barger, A. J., Cowie, L. L., Mushotzky, R. F., et al. 2005, AJ, 129, 578
Bower, R. G., Hasinger, G., Castander, F. J., et al. 1996, MNRAS, 281, 59

Boyle, B. J., Griffiths, R. E., Shanks, T., Stewart, G. C., \& Georgantopoulos, I. 1993, MNRAS, 260, 49

Comastri, A., Setti, G., Zamorani, G., \& Hasinger, G. 1995, A\&A, 296, 1

Cowie, L. L., Barger, A. J., Hu, E. M., Capak, P., \& Songaila, A. 2004, AJ, 127, 3137

Croom, S. M., Smith, R. J., Boyle, B. J., et al. 2004, MNRAS, 349, 1397

Dickinson, M., et al. 2003 in The Mass of Galaxies at low and high redshift, ed. R. Bender, \& A. Renzini (New York: Springer), 324

Duschl, W. J., \& Strittmatter, P. A. 2002, in Active Galactic Nuclei: from Central Engine to Host Galaxy Abstract Book, meeting held in Meudon, France, July 23-27, 2002, ed. S. Collin, F. Combes, \& I. Shlosman. To be published in ASP Conf. Ser., 76

Fadda, D., Flores, H., Hasinger, G., et al. 2002, A\&A, 383, 838

Fan, X., Strauss, M., Richards, G., et al. 2001, AJ, 121, 31

Fasano, G., \& Franceschini, A. 1987, MNRAS, 225, 155

Fiore, F., Brusa, M., Cocchia, F., et al. 2003, A\&A, 409, 79

Franceschini, A., Hasinger, G., Miyaji, T., \& Malquori, D. 1999, MNRAS, 310, L5

Franceschini, A., Braito, V., \& Fadda, D. 2002, MNRAS, 335, L51

Gehrels, N. 1986, ApJ, 303, 336

Giacconi, R., Rosati, P., Tozzi, P., et al. 2001, ApJ, 551, 624

Giacconi, R., Zirm, A., Wang, J. X., et al. 2002, ApJS, 139, 369

Giavalisco, M., Ferguson, H. C., Koekemoer, A. M., et al. 2004, ApJ, 600, L93

Gilli, R., Cimatti, A., Daddi, E., et al. 2003, ApJ, 592, 721

Gilli, R., Daddi, E., Zamorani, G., et al. 2005, A\&A, 430, 811

Gioia, I., Henry, J. P., Mullis, C. R., et al. 2003, ApJS, 149, 29

Hasinger, G., Burg, R., Giacconi, R., et al. 1993, A\&A, 275, 1

Hasinger, G., Burg, R., Giacconi, R., et al. 1998, A\&A, 329, 482

Hasinger, G., Altieri, B., Arnaud, M., et al. 2001, A\&A, 365, 45

Hasinger, G. 2004, Nucl. Phys. B (Proc. Suppl.), 132, 86

Hornschemeier, A. E., Brandt, W. N., Garmire, G. P., et al. 2000, ApJ, 541

Jones, L. R., McHardy, I. M., Merrifield, M. R., et al. 1997, MNRAS, 285,547

Kauffmann, G., \& Haehnelt, M. 2000, MNRAS, 311, 576

Kinney, A. L. 1996, ApJ, 467, 38

Koekemoer, A. M., Alexander, D. M., Bauer, F. E., et al. 2004, ApJ, 600, L123

Lehmann, I., Hasinger, G., Schmidt, M., et al. 2000, A\&A, 354, 35

Lehmann, I., Hasinger, G., Schmidt, M., et al. 2001, A\&A, 371, 833

Madau, P., Pozzetti, L., \& Dickinson, M. 1998, ApJ, 498, 106

Mainieri, V., Bergeron, J., Rosati, P., et al. 2002, A\&A, 393, 425

Mainieri, V., Rosati, P., Tozzi, P., et al. 2005, A\&A, 437, 805

McHardy, I. M., Jones, L. R., Merrifield, M. R., et al. 1998, MNRAS, 295, 641

Merloni, A. 2004, MNRAS, 353, 1035

Miyaji, T., Hasinger, G., \& Schmidt, M. 2000, A\&A, 353, 25 (Paper I)

Miyaji, T., Hasinger, G., \& Schmidt, M. 2001, A\&A, 369, 49 (Paper II)

Mobasher, B., Idzi, R., Benitez, N., et al. 2004, ApJ, 600, L167

Moran, E. C., Filippenko, A. V., \& Chornock, R. 2000, ApJ, 579, L71

Moretti, A., Campana, S., Lazzati, D., et al. 2003, ApJ, 588, 696

Mullis, C. R., Henry, J. P., Gioia, I. M., et al. 2004, ApJ, 617, 192

Mushotzky, R. F., Cowie, L. L., Barger, A. J., \& Arnaud, K. A. 2000, Nature, 404, 459

Norman, C., Ptak, A., Hornschemeier, A., et al. 2004, ApJ, 607, 721

Mason, K. O., Carrera, F. J., Hasinger, G., et al. 2000, MNRAS, 311, 456 
Page, M. J., Mason, K. O., McHardy, I. M., Jones, L. R., \& Carrera, F. J. 1997, MNRAS, 291, 324

Press, W. H., Teukolsky, S. A., Vetterling, W. T., \& Flannery, B. P. 1992, Numerical Recipes in Fortran (Cambridge: Cambridge Univ. Press), 640

Rosati, P., Tozzi, P., Giacconi, R., et al. 2002, ApJ, 566, 667

Salvato, M. 2002, Dissertation, Potsdam University

Schmidt, M. 1968, ApJ, 151, 393

Schmidt, M., \& Green, R. F. 1983, ApJ, 269, 352

Schmidt, M., Schneider, D. P., \& Gunn, J. E. 1995, AJ, 110, 68

Schmidt, M., Hasinger, G., Gunn, J. E., et al. 1998, A\&A, 329, 495

Schwope, A., Hasinger, G., Lehmann, I., et al. 2000, AN, 321, 1

Silverman, J. D., Green, P. J., Barkhouse, W. A., et al. 2004, ApJ, 624, 630

Spergel, D. N., Verde, L., Peiris, H. V., et al. 2003, ApJS, 148, 175

Steffen, A. T., Barger, A. J., Capak, P., et al. 2004, AJ, 128, 1483
Szokoly, G., Bergeron, J., Hasinger, G., et al. 2004, ApJS, 155, 271

Ueda, Y., Akiyama, M., Ohta, K., \& Miyaji, T. 2003, ApJ, 598, 886

Vignali, C., Brandt, W. N., Schneider, D. P., et al. 2003, AJ, 125, 2876

Voges, W., Aschenbach, B., Boller, Th., et al. 1999, A\&A, 349, 389

Wall, J. V., Jackson, C. A., Shaver, P. A., Hook, I. M., \& Kellermann, K. I. 2005, A\&A, 434, 133

Wolf, C., Meisenheimer, K., Kleinheinrich, M., et al. 2004, A\&A, 421, 913

Worsley, M., Fabian, A. C., Mateos, S., et al. 2004, MNRAS, 352, L28

Worsley, M., Fabian, A. C., Bauer, F., et al. 2005, MNRAS, 357, 1281

Wyithe, J. S. B., \& Loeb, A. 2003, ApJ, 595, 614

Yang, Y., Mushotzky, R. F., Steffen, A. T., Barger, A. J., \& Cowie, L. L. 2004, AJ, 128, 1501

Zamorani, G., Mignoli, M., Hasinger, G., et al. 1999, A\&A, 346, 731

Zheng, W., Mikles, V. J., Mainieri, V., et al. 2004, ApJS, 155, 73 